\title{
A Robust Fractional Model for Improving Industrial Park Water Use Efficiency Under Dual Uncertainties
}

\section{Zhuoling Lan}

Guangdong University of Technology - University Town Campus: Guangdong University of Technology

\section{Shuping Wang}

China Agricultural University

Qian Tan ( $\nabla$ qian_tan@cau.edu.cn )

Guangdong University of Technology - University Town Campus: Guangdong University of Technology

Tianyuan Zhang

China Agricultural University

\section{Yini Cao}

Guangdong University of Technology - University Town Campus: Guangdong University of Technology

\section{Research Article}

Keywords: linear partial information, two-stage stochastic programming, fractional programming, industrial park water management, dual uncertainties

Posted Date: February 16th, 2022

DOI: https://doi.org/10.21203/rs.3.rs-1356723/v1

License: (c) (1) This work is licensed under a Creative Commons Attribution 4.0 International License.

Read Full License 


\section{A robust fractional model for improving industrial park}

2 water use efficiency under dual uncertainties

3 Zhuoling Lan ${ }^{\mathrm{a}}$, Shuping Wang ${ }^{\mathrm{b}}$, Qian Tan ${ }^{\mathrm{a}, \mathrm{b}, *}$, Tianyuan Zhang ${ }^{\mathrm{b}}$, Yini Cao ${ }^{\mathrm{a}}$

$4 \quad{ }^{a}$ Key Laboratory for City Cluster Environmental Safety and Green Development of

5 the Ministry of Education, Institute of Environmental and Ecological Engineering,

6 Guangdong University of Technology, Guangzhou 510006, China

7 bollege of Water Resources and Civil Engineering, China Agricultural University,

$8 \quad$ Beijing 100083, China

9 Abstract:

As an existing form of high aggregation of industries, industrial parks, have problems with a large demand for water and sewage discharge. Therefore, developing an effective approach to improve the efficiency of industrial park water use is urgently needed. Originating from internal and external changes, complicated uncertainties may coexist in multiple system components and their correlations within industrial park water resource management. Previous studies ignored the uncertain probability related to water inflow, which is difficult to estimate for long-term decision-making problems. This research describes the exploitation of an inexact two-stage stochastic partial fractional programming (ITSPF) method for sustainable industrial park water supply, under dual uncertainties. ITSPF is developed via the synthesis of linear partial information, interval programming, two-stage stochastic programming, and fractional programming techniques. It could improve conventional industrial park water resource optimization by solving the uncertain probability of water inflow levels while optimizing the ratio issues. The results show that various water allocation plans within different inflow level probabilities can be generated by handling the trade-off between water consumption and system benefit. And the amount of reclaimed water 
would be increased under the higher risk of water shortage. The comparisons of ITSPF results against the least-cost model and model with deterministic probability of water inflow levels demonstrated that ITSPF could not only result in higher resource-use efficiency, but also avoid missing possible solution sets and offer a pragmatic way for obtaining satisfactory alternatives by providing wider adjustable ranges.

Keywords: linear partial information, two-stage stochastic programming, fractional programming, industrial park water management, dual uncertainties

\section{Highlights}

- An inexact two-stage stochastic partial fractional (ITSPF) model was developed.

- It may handle dual uncertainties and ratio issues for industrial park water system.

- Various schemes under different probability of inflow levels were generated.

- Comparative cases were considered to validate the superiority of ITSPF.

\section{Introduction}

Global industrial water withdrawals are predicted to approach $2000 \mathrm{~km}^{3} / \mathrm{yr}$ by 2050 , more than double the industrial water consumption in $2018\left(920 \mathrm{~km}^{3} / \mathrm{yr}\right)$ (Burek et al., 2016; Wada et al., 2016; Boretti and Rosa, 2019; Bauer et al., 2020). The sustainability of industrial activities depends on the water availability, cost, and quality (WBCSD, 2006; Boysen et al., 2020). As an existing form of high aggregation of industries, industrial parks can obtain higher economic benefits, but have problems with large demands for water and sewage discharge. Among industrial park water-using networks, the case is usually treated as a water distribution issue through a superstructure-based model where water needs to be allocated, treated, and discharged optimally between the process units of various enterprises (Boix et al., 2015; Lawal et al., 2021). In industrial park water management systems, the decision 
makers need to consider many complex processes because of the competition between economic development and environmental protection. The periodical decisions in industrial park water management issues are required with the passage of time, resulting in a wide array of dynamic complexities. Furthermore, plenty of uncertainties may coexist in multiple system components and their correlations within an industrial park water management (Boix et al., 2012). These uncertainties may stem from inner and outer interactions in water systems, such as variations in technical or commercial parameters cause by technological advances, fluctuations in water requirements cause by changing society and ecological systems, and shifts in water-circulation patterns cause by climate change (Tan et al., 2007, 2010; Chew et al., 2009; Tan et al., 2013; Khor et al., 2014; Bi et al., 2019). Thus, efficient optimization tools for planning industrial park water resource systems under these complexities are desired (Wang et al., 2016).

Over the past few decades, numerous studies have rapidly developed in the field of industrial park water resource allocation optimization, which is generally optimized through pinch technology and mathematical programming optimization (Yoo et al., 2007; Boix et al., 2015; Kastner et al., 2015). The pinch analysis provides easy-to-understand water allocated solutions due to its graphical concepts (Olesen and Polley, 1996; El-Halwagi et al., 2003). Compared to pinch analysis, mathematical programming approaches are well-adapted to solve large-scale and complex issues (e.g., multi-period water exchange (Liao et al., 2007), multi-objective optimization (Tiu and Cruz, 2017), multi-contaminant problems (Boix et al., 2011), etc.), which have been well studied in the literature with the concept of superstructure (Boix et al., 2015). Typically, practical industrial park water resource systems are complex because of the conflicts among enterprises, natural resources along with social, 
economic, and ecological factors. It is obvious that the optimal water allocation of an industrial park through single-objective optimization is trivial (Ramos et al., 2016). Multi-objective programming (MOP) approaches are useful to tackle these pressing problems (Montastruc et al., 2013). In most applications, plenty of algorithms are taken to explore the "most preferred" solution in industrial park water optimization to circumvent the drawbacks of conventional linear programming approaches. (Mavrotas, 2009). One is converting multi-objective problems into single-objective problems (Tiu and Cruz, 2017). The other is to obtain efficient solutions to the problem through the Pareto front (Boix et al., 2011; 2012). The former algorithm requires subjective and unrealistic assumptions to transform multiple conflicting objectives into a single monetary measure. (Marler and Arora, 2010; Zhu et al., 2014; Ji et al., 2017). The latter has a large computational effort and a lack of widely available software (Mavrotas, 2009). Finally, MOP methods may encountered difficulties in considering and assessing ratio issues, although it can balance conflicting objectives and coordinate the interests of different stakeholders (Zhu and Huang, 2011).

Fractional programming (FP) can deal with conflict objective expressed as ratio problem without considering subjective information, which can objectively avoid the weaknesses of MOP methods (Stancu-Minasian, 1997). It is considered to be an effective tool for ratio optimization, which can offer an unprejudiced method of system efficiency and optimize marginal system benefits effectively (Mehra et al., 2007; Zhu and Huang, 2013; Cui et al., 2015; Zhou et al., 2015; Zhou et al., 2016; Tan and Zhang, 2018). In addition, the objective function in FP is the quotient of physical and economic quantities which is proven to be a highly effective measure to meet the needs of real-world problems in resource management, finance, transportation, and production. A classic algorithm for FP was first developed by Charnes and Cooper 
101 (1963) and the latest progress has been reported as well in Stancu-Minasian (2019).

FP has been well studied in measuring the efficiency of water resource systems (Fasakhodi et al., 2010; Mani et al., 2016; Zhao et al., 2017). Nevertheless, this method has seldom been applied to address industrial park water management problems. Moreover, the uncertainties that are prevalent in industrial park water resource management cannot be veritably resolved in previous FP studies.

Industrial park water management problems contain many uncertain factors and inexact parameters which cannot be expressed accurately. Hence, many optimization methods have been proposed to address these uncertainties (Li et al., 2008; Aviso et al., 2010). Particularly, a large amount of researches drawing great attention to two-stage stochastic programming (TSP), which has been widely adopted for solving combined uncertainties and economic consequences of violating the committed targets in water resource management issues (Huang and Loucks, 2000; Xie et al., 2013; Zhou et al., 2015). It is considered an efficient approach for dealing with uncertainties expressed as probabilities, where an analysis of multi-stage decisions is desired to take recourse or for corrective actions (Khor et al., 2014; Cui et al., 2015; Zhou et al., 2015). But the existing TSP-based methods only address inflow probabilities, which are known as deterministic values. In industrial park water management problems, the probabilistic information of water inflow may be partially determined (Kofler, 2001). In practice, the water inflow is usually estimated by the number of random events within a certain period of time. Different water resource monitoring sectors have observation stations themselves to collect water inflow data. Therefore, the probability density functions of the future stream flow will be varied, which results in some uncertainties in the probability of the water inflow. Such compound uncertainties can be solved by introducing linear partial information (LPI), 
in which the probability of water inflow levels fluctuates within a certain range and is expressed as an interval (Fan et al., 2012b; Chen and Wang, 2021). However, there is no further study addressing the dual uncertainties with FP, which can optimize the water use efficiency in industrial park water resources management.

Therefore, this research develop an inexact two-stage stochastic partial fractional (ITSPF) programming to tackle dual uncertainties and ratio issues involved in industrial park water resource management problems. Fractional programming is first incorporated with inexact two-stage stochastic partial programming to address multi-objective issues. The ITSPF method can balance two conflict aspects expressed as ratio issues and analyze various scenarios under corresponding probability distributions of water inflows. Thus, it can assist planners in identifying the optimal strategies of industrial park water resource management and gain in-depth insights into water allocation pattern, system failure risk, and system profit under different scenarios. A representative industrial park water resource management system is developed to further demonstrate the applicability of the developed method.

\section{Complexities of industrial park water resources system}

At present, the availability of water is critical for industrial park as the water demands for factories are increasing dramatically for their own development. For industrial park, surface water and underground water are considered as its potential sources. Underground water is regarded as an important water resource because of the stable quality and quantity. Overdraft of groundwater may cause adverse natural phenomena such as land subsidence, soil salinization, seawater intrusion, and catastrophic damages to ecological systems. Thus the exploitation of underground water is limited (Tan et al., 2013). The quality of surface water would lower than underground water due to its widely exposition to point and non-point pollution sources. Despite the 
abundance of surface water, the severe contamination of it may be highly undesirable to water supply. Above all, the availability of water sources are limited cause by the polluted surface water and over exploitation of underground water. This will result in various kinds of negative impacts on the sustainable development of social-economic and ecological environment. Thus, the supplement of reclaimed water is desired which can facilitate the reduction of fresh water consumption. Reclaimed water is wastewater that has been properly treated by removing solids and impurities. The capacity of regeneration unit and the efficiency of sewage collection system are important factors for the availability of reclaimed water. Schematically, the water allocation pattern for an industrial park can be presented in Fig.1. For each water user plants, input water may be surface water, underground water and their own reclaimed water; the output water for each plant may be distributed to regeneration units or wastewater treatment plant.

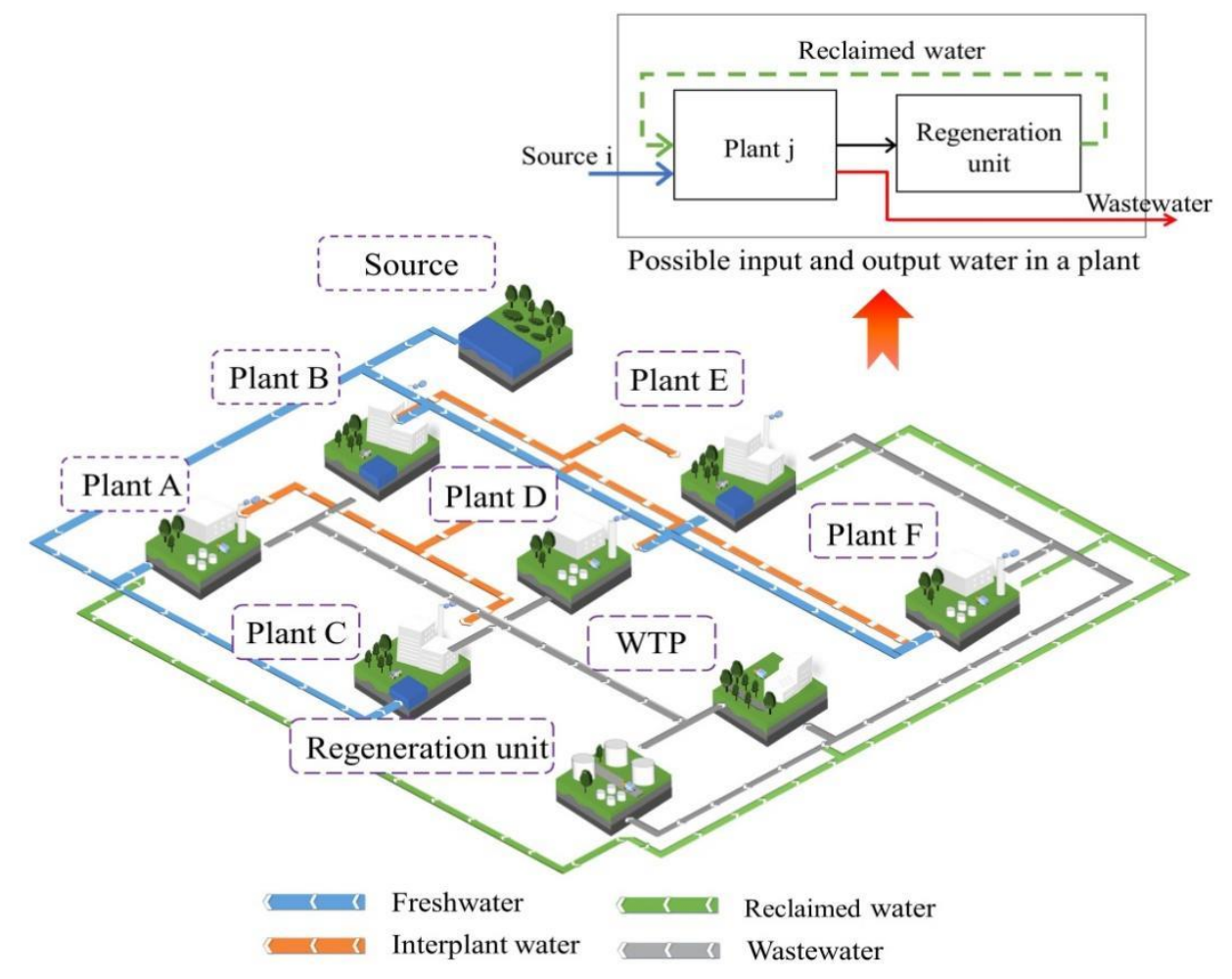

Fig.1 Possible connections in an industrial park (WTP represents the wastewater treatment plant) In the meantime, a great deal of conflicting goals, interactions and dynamics may 
coexist within industrial park (Taskhiri et al., 2011). In industrial park water resources management problem, the amount of available water resources have random feature because of the temporal variation of water resources system. This will affect the optimal water resource management in industrial park. TSP can address uncertainties expressed as probabilistic distributions effectively (Huang and Loucks, 2000; Xie et al., 2013). But the existing TSP-based methods only address the inflow probabilities is known as deterministic values, which ignored the uncertain probability of water inflow level. By introducing LPI into TSP, the uncertain probability of water inflow levels would fluctuate within a certain range and express as interval, which is helpful to deal with the dual uncertainties related to random inflows in water resources management. According to the partial distribution probability mentions in equation (2.1), the solution of the inequality (1.7) is defined as extreme points. Therefore the results of extreme points $\mathrm{p}_{1}, \mathrm{p}_{2}$ and $\mathrm{p}_{3}$ would take the interval values of $[0.1,0.2],[0.5$, $0.7]$ and $[0.2,0.3]$, respectively (Fan et al., 2012b). Due to the sum of these extreme points is equal to 1, there are four combinations presented by A, B, C, D in Fig.2. Until now, the formulation of existed MOP methods for industrial park water management systems need to be converted into single objective problems or obtained the efficient solutions of the problem through Pareto front which is susceptible to subjective factors and large computational efforts. The essence of fractional programming is a ratio optimization problem, which can coordinate conflicting objectives and obtain the optimal scheme to improve system efficiency. Besides, multiple interval parameters, such as water availability, water demands, and operational costs, will further amplified the system complexities. Therefore, an inexact two-stage stochastic partial fractional industrial park water resource management (ITSPF-IPWRM) model based on the ITSPF method is desired to assist 
193

$$
\max f^{ \pm}=\frac{\sum_{j=1}^{n} c_{j}^{ \pm} x_{j}^{ \pm}+\sum_{h=1}^{q} \sum_{j=1}^{n} p_{h} e_{j}^{ \pm} y_{j h}^{ \pm}+\alpha^{ \pm}}{\sum_{j=1}^{n} d_{j}^{ \pm} x_{j}^{ \pm}+\sum_{h=1}^{q} \sum_{j=1}^{n} p_{h} g_{j}^{ \pm} y_{j h}^{ \pm}+\beta^{ \pm}}
$$

Fig.2 Partial probability distribution of inflow (Fan et al., 2012b)

\section{Development of the ITSPF model}

\subsection{Methodology}

A particular type of stochastic partial information - LPI would be introduced to express the probability distribution as interval, which can solve the uncertainties of the probabilistic information to water inflow. Thus, an ITSPF method which combine interval programming, linear partial information, two-stage stochastic programming and fractional programming techniques will be proposed to address uncertain probability distribution described as interval parameters while measuring conflict objectives. The general ITSPF model can be calculated as follows :

subject to:

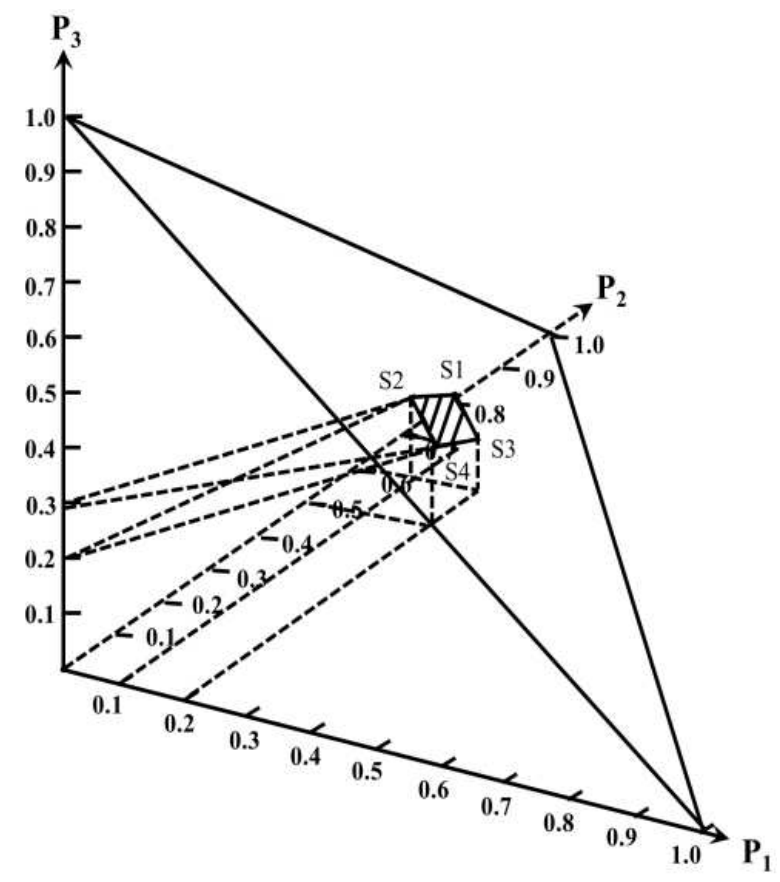


$206 \quad \sum_{j=1}^{n} a_{k j}^{ \pm} x_{j}^{ \pm}+\sum_{j=1}^{n} u_{k j}^{ \pm} y_{j h}^{ \pm} \leq b_{k}^{ \pm}, k=1,2, \mathrm{~L}, p ; h=1,2, \mathrm{~L}, q$

$207 \quad \sum_{j=1}^{n} a_{i j}^{ \pm} x_{j}^{ \pm}+\sum_{j=1}^{n} u_{i j}^{ \pm} y_{j h}^{ \pm} \geq w_{i h}^{ \pm}, i=1,2, \mathrm{~L}, I ; h=1,2, \mathrm{~L}, q$

$208 \quad x_{j}^{ \pm} \geq 0, j=1,2, \mathrm{~L}, t, r+1, \mathrm{~L}, r+l$

$209 y_{j h}^{ \pm} \geq 0, j=1,2, \mathrm{~L}, t, r+1, \mathrm{~L}, r+l ; h=1,2, \mathrm{~L}, q$

$210 \quad \sum_{h=1}^{q} p_{h}=1$

$211 H P \leq b$

$212 \quad p_{h} \geq 0$

213 Where $x_{j}^{ \pm}$is the first-stage variable and $y_{j h}^{ \pm}$is the second-stage variable; the

214 coefficients of first-stage variables are $a_{k j}^{ \pm}, c_{j}^{ \pm}, d_{j}^{ \pm}$and $a_{i j}^{ \pm}$, and the coefficients of

215 second-stage variables are $e_{j}^{ \pm}, g_{j}^{ \pm}, u_{k j}^{ \pm}$and $u_{i j}^{ \pm}, \alpha^{ \pm}$and $\beta^{ \pm}$are constants, and $b_{k}^{ \pm}$

216 are right-hand-side parameters, $w_{i h}^{ \pm}$is random right-hand-side parameters of

217 constraint $k$ under different probability levels (Lin and Huang, 2009). Equations (1.4)

218 to (1.5) represent the non-negative decision variables.

219 The equations (1.6) to (1.7) represent the constraint of partial information. And the 220 detailed partial probability distribution can be expressed as equation (2.1) (Kofler, 221 2001). Firstly, the probability $p_{j}$ is fluctuate within $\delta$ and $\varepsilon$, where $\delta$ and $\varepsilon$ 222 are fixed numbers (i.e., $\delta \leq p_{h} \leq \varepsilon$ ). Moreover, the probability of $p_{j}$ and $p_{i}$ is 223 equal to $\gamma$ (i.e., $p_{i}+p_{h}=\gamma$ ). The information mention above can be shown as 224 followings(Kofler, 2001):

225

$$
\pi=\left\{P=\left(p_{1}, \mathrm{~L}, p_{N}\right) \in R^{N} \text { s.t. } H P \leq b, \sum_{h=1}^{N} p_{h}=1 ; p_{h} \geq 0, h=1, \mathrm{~L} N\right\}
$$


where $H=\left(h_{i j}\right)$ and $b=\left(b_{i}\right)$ are $s \times N$ and $s \times l$ fixed matrices, respectively. The partial distribution probability described as equation (2.1) can effectively address uncertain probability issues. When the $p_{1}^{*}, \mathrm{~L}, p_{n}^{*}$ satisfy $n$ of the restrictions contained in $H P \leq b$ as independent equations and the restriction $\sum_{h=1}^{N} p_{h}=1$ is eligible among these $n$ equations, a solution $P^{*}=\left(p_{1}^{*}, \mathrm{~L}, p_{n}^{*}\right)$ of the inequality (1.7) is an extreme point (Kofler and Zweifel, 1991). Meanwhile, the expected value of a random variable $X$ expressed as equation (2.1) can be defined within the expected interval $\left[E_{\min }(X), E_{\max }(X)\right] \quad, \quad$ where $\quad E_{\min }(x)=\min _{\left\{p^{*}\right\}} \sum_{h=1}^{n} p_{j} x_{j} \quad$ and $E_{\max }(x)=\max _{\left\{p^{*}\right\}} \sum_{j=1}^{n} p_{j} x_{j}$. Therefore, ITSPF model would get its interval values when the probabilities inflow $q_{j}$ reach the extreme points by constraints (1.6) to

ITSPF can be addressed by a scenario based interactive algorithm where two deterministic sub-models are converted to get its lower and upper bounds. Firstly, extreme points of the linear partial information (LPI) will be obtained by constraints (1.6) to (1.8). Assuming $P^{l^{*}}=\left(p_{1}^{l^{*}}, \mathrm{~L}, p_{n}^{l *}\right) \quad(l=1, \ldots, r)$ are the extreme points

241 determined by equations (1.6) to (1.8). For one $P^{l}$, model (1) can convert to an 242 inexact two-stage fractional (ITF) approach calculated as follows:

$$
\max f^{l \pm}=\frac{\sum_{j=1}^{n} c_{j}^{ \pm} x_{j}^{ \pm}+\sum_{h=1}^{q} \sum_{j=1}^{n} p_{h}^{l^{*}} e_{j}^{ \pm} y_{j h}^{l \pm}+\alpha^{ \pm}}{\sum_{j=1}^{n} d_{j}^{ \pm} x_{j}^{ \pm}+\sum_{h=1}^{q} \sum_{j=1}^{n} p_{h}^{l^{*}} g_{j}^{ \pm} y_{j h}^{l \pm}+\beta^{ \pm}}
$$

244 subject to:

$$
\sum_{j=1}^{n} a_{k j}^{ \pm} x_{j}^{ \pm}+\sum_{j=1}^{n} u_{k j}^{ \pm} y_{j h}^{l \pm} \leq b_{k}^{ \pm}, k=1,2, \mathrm{~L}, p ; h=1,2, \mathrm{~L}, q
$$


$246 \quad \sum_{j=1}^{n} a_{i j}^{ \pm} x_{j}^{ \pm}+\sum_{j=1}^{n} u_{i j}^{ \pm} y_{j h}^{l \pm} \geq w_{i h}^{ \pm}, i=1,2, \mathrm{~L}, I ; h=1,2, \mathrm{~L}, q$

$247 \quad x_{j}^{ \pm} \geq 0, j=1,2, \mathrm{~L}, t, r+1, \mathrm{~L}, r+l$

$248 y_{j h}^{l \pm} \geq 0, j=1,2, \mathrm{~L}, t, r+1, \mathrm{~L}, r+l ; h=1,2, \mathrm{~L}, q$

249 Secondly, model (3) can be converted to two sub-models by interactive algorithm

250 (Hladik, 2010). Since the decision variables are independent, the interactions between

251 the bounds of $x_{j}^{ \pm}$(or $y_{j}^{ \pm}$) and $f^{ \pm}$can be formulated by Zhu et al. (2014). To

252 acquire solutions more precisely, sub-model corresponding to $f^{-}$is formulated first

253 (Fan and Huang, 2012a):

$254 \max f^{l-}=\frac{\sum_{j=1}^{r} c_{j}^{-} x_{j}^{-}+\sum_{j=r+1}^{n} c_{j}^{-} x_{j}^{+}+\sum_{h=1}^{q} \sum_{j=1}^{r} p_{h}^{l^{*}} e_{j}^{-} y_{j h}^{l-}+\sum_{h=1}^{q} \sum_{j=r+1}^{n} p_{h}^{l^{*}} e_{j}^{-} y_{j h}^{l+}+\alpha^{-}}{\sum_{j=1}^{r} d_{j}^{+} x_{j}^{-}+\sum_{j=r+1}^{n} d_{j}^{+} x_{j}^{+}+\sum_{h=1}^{q} \sum_{j=1}^{r} p_{h}^{l^{*}} g_{j}^{+} y_{j h}^{l-}+\sum_{h=1}^{q} \sum_{j=r+1}^{n} p_{h}^{l^{*}} g_{j}^{+} y_{j h}^{l+}+\beta^{+}}$

255 subject to:

$256 \sum_{j=1}^{r}\left|a_{k j}^{ \pm}\right|^{+} \operatorname{sign}\left(a_{k j}^{ \pm}\right) x_{j}^{-}+\sum_{j=r+1}^{n}\left|a_{k j}^{ \pm}\right|^{-} \operatorname{sign}\left(a_{k j}^{ \pm}\right) x_{j}^{+}+\sum_{j=1}^{r}\left|u_{k j}^{ \pm}\right|^{+} \operatorname{sign}\left(u_{k j}^{ \pm}\right) y_{j h}^{l-}+\sum_{j=r+1}^{n}\left|u_{k j}^{ \pm}\right|^{-} \operatorname{sign}\left(u_{k j}^{ \pm}\right) y_{j h}^{l+} \leq b_{k}^{-}$

$257 k=1,2, \mathrm{~L}, p ; h=1,2, \mathrm{~L}, q$

$258 \sum_{j=1}^{r}\left|a_{i j}^{ \pm}\right|^{+} \operatorname{sign}\left(a_{i j}^{ \pm}\right) x_{j}^{-}+\sum_{j=r+1}^{n}\left|a_{i j}^{ \pm}\right|^{-} \operatorname{sign}\left(a_{i j}^{ \pm}\right) x_{j}^{+}+\sum_{j=1}^{r}\left|u_{i j}^{ \pm}\right|^{+} \operatorname{sign}\left(u_{i j}^{ \pm}\right) y_{j h}^{l-}+\sum_{j=r+1}^{n}\left|u_{i j}^{ \pm}\right|^{-} \operatorname{sign}\left(u_{i j}^{ \pm}\right) y_{j h}^{l+} \geq w_{i h}^{+}$

$259 \quad i=1,2, \mathrm{~L}, I ; h=1,2, \mathrm{~L}, q$

$260 \quad x_{j}^{-} \geq 0, j=1,2, \mathrm{~L}, t$

261

$x_{j}^{+} \geq 0, j=r+1, \mathrm{~L}, r+l$

262

$y_{j h}^{l-} \geq 0, j=1,2, \mathrm{~L}, t ; h=1,2, \mathrm{~L}, q$

263

$y_{j h}^{l+} \geq 0, j=r+1, \mathrm{~L}, r+l ; h=1,2, \mathrm{~L}, q$

$264 \quad \operatorname{sign}\left(a_{i j}^{ \pm}\right)=\left\{\begin{array}{rrr}1 & \text { if } & a_{i j}^{ \pm} \geq 0 \\ -1 & \text { if } & a_{i j}^{ \pm} \leq 0\end{array}\right.$ 
$265 \operatorname{sign}\left(a_{k j}^{ \pm}\right), \operatorname{sign}\left(u_{i j}^{ \pm}\right)$and $\operatorname{sign}\left(u_{k j}^{ \pm}\right)$can be interpreted similarly. $\left|a_{i j}^{ \pm}\right|^{ \pm}$is defined as:

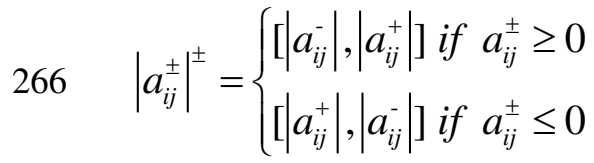

$267\left|a_{k j}^{ \pm}\right|^{ \pm},\left|u_{i j}^{ \pm}\right|^{ \pm}$and $\left|u_{k j}^{ \pm}\right|^{ \pm}$can be interpreted similarly.

268 The solutions of $x_{j o p t}^{-}(j=1,2, \ldots, r), x_{j o p t}^{+}(j=r+1, \ldots, n), \quad y_{\text {jhopt }}^{l-}(j=1,2, \ldots, r)$ and $269 y_{\text {jhopt }}^{l+}(j=r+1, \ldots, n)$ can be obtained from the sub-model mention above. Then the 270 interactive sub-model corresponding to $f^{+}$can be described as follows:

$$
\max f^{l+}=\frac{\sum_{j=1}^{r} c_{j}^{+} x_{j}^{+}+\sum_{j=r+1}^{n} c_{j}^{+} x_{j}^{-}+\sum_{h=1}^{q} \sum_{j=1}^{r} p_{h}^{l^{*}} e_{j}^{+} y_{j h}^{l+}+\sum_{h=1}^{q} \sum_{j=r+1}^{n} p_{h}^{l^{*}} e_{j}^{+} y_{j h}^{l-}+\alpha^{+}}{\sum_{j=1}^{r} d_{j}^{-} x_{j}^{+}+\sum_{j=r+1}^{n} d_{j}^{-} x_{j}^{-}+\sum_{h=1}^{q} \sum_{j=1}^{r} p_{h}^{l^{*}} g_{j}^{-} y_{j h}^{l+}+\sum_{h=1}^{q} \sum_{j=r+1}^{n} p_{h}^{l^{*}} g_{j}^{-} y_{j h}^{l-}+\beta^{-}}
$$

272 subject to:

$273 \sum_{j=1}^{r}\left|a_{k j}^{ \pm}\right|^{-} \operatorname{sign}\left(a_{k j}^{ \pm}\right) x_{j}^{+}+\sum_{j=r+1}^{n}\left|a_{k j}^{ \pm}\right|^{+} \operatorname{sign}\left(a_{k j}^{ \pm}\right) x_{j}^{-}+\sum_{j=1}^{r}\left|u_{k j}^{ \pm}\right| \operatorname{sign}\left(u_{k j}^{ \pm}\right) y_{j h}^{l+}+\sum_{j=r+1}^{n}\left|u_{k j}^{ \pm}\right|^{+} \operatorname{sign}\left(u_{k j}^{ \pm}\right) y_{j h}^{l-} \leq b_{k}^{+}$

$274 k=1,2, \mathrm{~L}, p ; h=1,2, \mathrm{~L}, q$

$275 \sum_{j=1}^{r}\left|a_{i j}^{ \pm}\right|^{-} \operatorname{sign}\left(a_{i j}^{ \pm}\right) x_{j}^{+}+\sum_{j=r+1}^{n}\left|a_{i j}^{ \pm}\right|^{+} \operatorname{sign}\left(a_{i j}^{ \pm}\right) x_{j}^{-}+\sum_{j=1}^{r}\left|u_{i j}^{ \pm}\right| \operatorname{sign}\left(u_{i j}^{ \pm}\right) y_{j h}^{l+}+\sum_{j=r+1}^{n}\left|u_{i j}^{ \pm}\right|^{+} \operatorname{sign}\left(u_{i j}^{ \pm}\right) y_{j h}^{l-} \geq w_{i h}^{-}$

$276 i=1,2, \mathrm{~L}, I ; h=1,2, \mathrm{~L}, q$

$277 \quad x_{j}^{+} \geq 0, j=1,2, \mathrm{~L}, t$

$278 x_{j}^{-} \geq 0, j=r+1, \mathrm{~L}, r+l$

$279 y_{j h}^{l+} \geq 0, j=1,2, \mathrm{~L}, t ; h=1,2, \mathrm{~L}, q$

280

$y_{j h}^{l-} \geq 0, j=r+1, \mathrm{~L}, r+l ; h=1,2, \mathrm{~L}, q$

$281 x_{j}^{+} \geq x_{j o p t}^{-}, j=1,2, \mathrm{~L}, r$

282

$$
x_{j}^{-} \leq x_{j o p t}^{+}, j=r+1, \ldots, n
$$


285 Thirdly, the above interactive sub-models can be solved by Charnes and Cooper (1963)

286 directly (Appendix A). After that, repeat equations (4.1) to (5.1) for $l=1, \ldots, r$, and 287 different interval solutions for model (1) under corresponding extreme points can be 288 obtained. If all these requirements have been satisfied, a sets of optimal intervals can 289 be obtained as $f^{ \pm}=\left(f_{\text {opt }}^{l \pm}, f_{\text {opt }}^{2 \pm}, \ldots, f_{\text {opt }}^{r \pm}\right)$. The final optimal solution for model (1) can 290 be expressed as $f_{\text {opt }}^{ \pm}=\left[\min _{l}\left(f_{\text {opt }}^{l-}\right), \max _{l}\left(f_{\text {opt }}^{l+}\right)\right]$. The framework of ITSPF model is 291 summarized in Fig.3.

292

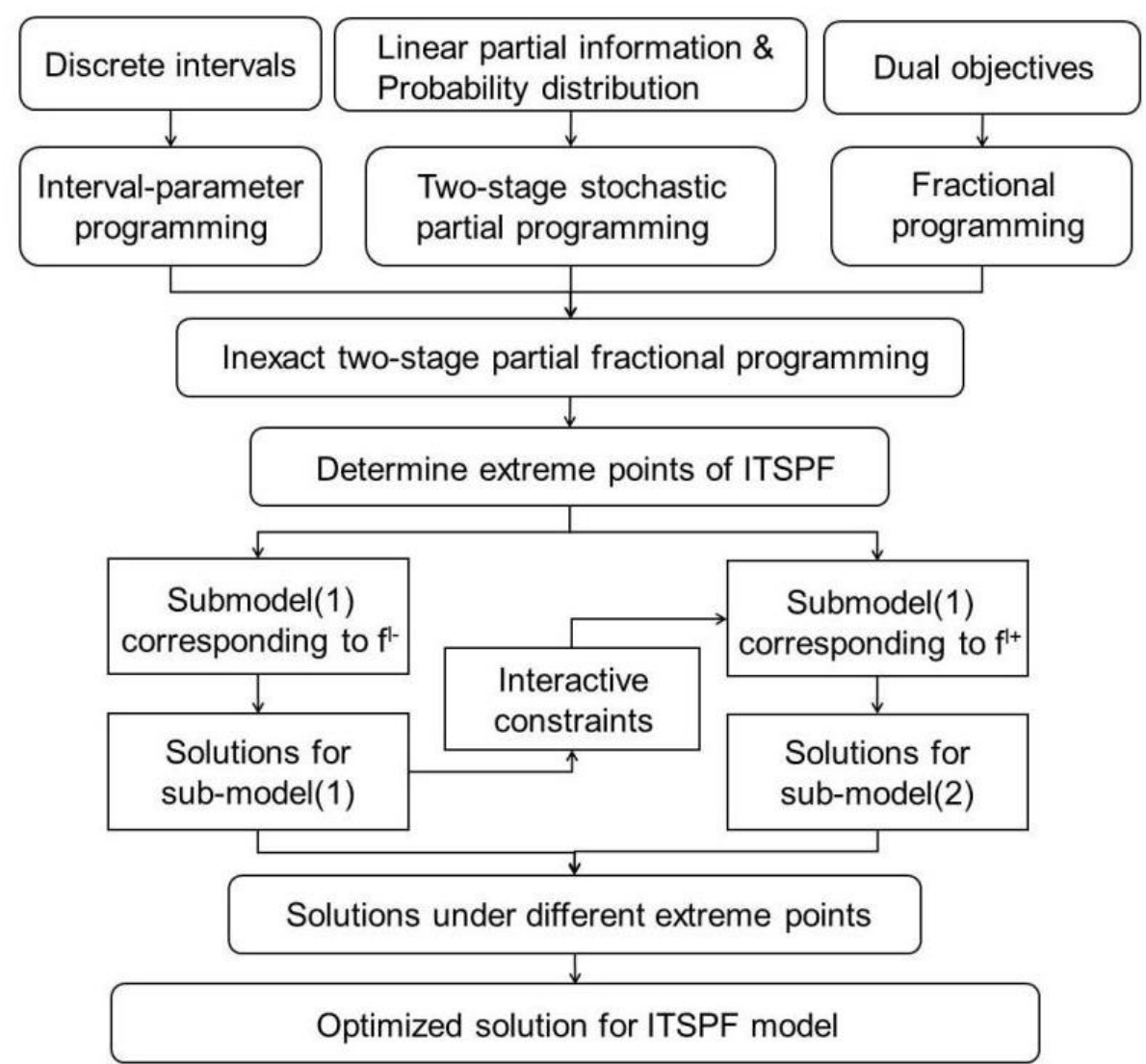

Fig.3 The framework of ITSPF model

\subsection{Model formulation}

In ITSPF-IPWRM, the goal is to maximize the economic efficiency of water 
consumption. Moreover, uncertain probability are addressed in constraint $(f)$. In detail, system net benefit of the ITSPF-IPWRM model can be described as follows:

298 (1) System net benefit

$$
f^{ \pm}{ }_{1}=\sum_{i=1}^{I} \sum_{j=1}^{J} \sum_{k=1}^{K} N B_{j k}^{ \pm} \times\left(T_{i j k}^{ \pm}+I R W_{j k}^{ \pm}\right)-\sum_{i=1}^{I} \sum_{j=1}^{N} \sum_{k=1}^{K} \sum_{h=1}^{H} p_{h}^{l^{*}} \times C T_{j k}^{ \pm} \times D_{i j k h}^{l \pm}
$$

where $i$ is the index for water sources; $j$ is the index for water user plants; $k$ is

301 index for planning periods; $h$ is the index for probability level; $N B_{j k}^{ \pm}$is net benefit

302 to user $j$ per unit of water allocated in period $k\left(y u a n / \mathrm{m}^{3}\right) ; T_{i j k}^{ \pm}$is fixed 303 allocation target for water that is promised from source $i$ to user $j$ in period $k$ $304\left(\mathrm{~m}^{3} /\right.$ day, the first-stage decision variable); $I R W_{j k}^{ \pm}$is the water supply targets during planning period $k$ from recycled water to user $j\left(\mathrm{~m}^{3} /\right.$ day); $C T_{j k}^{ \pm}$is reduction of net benefit to user $j$ per unit of source $i$ not delivered (yuan $/ \mathrm{m}^{3}, C T_{j k}^{ \pm}>N B_{j k}^{ \pm}$); $D_{i j k h}^{l \pm}$ is amount by which water allocation target $T_{i j k}^{ \pm}$is not met when the seasonal 308 flow is $q_{i h}^{ \pm}$with probability $p_{h}^{l^{*}}$ under extreme point $P^{l^{*}}\left(\mathrm{~m}^{3} /\right.$ day, the 309 second-stage decision variable).

310 (2) Costs for water supply

$311 \quad f_{2}^{ \pm}=\sum_{i=1}^{I} \sum_{j=1}^{J} \sum_{k=1}^{K} \sum_{h=1}^{H}\left(T_{i j k}^{ \pm}-D_{i j k h}^{l \pm} \times p_{h}^{l^{*}}\right) \times W S C_{j k}^{ \pm}$

312 where $W S C_{j k}^{ \pm}$is unit cost of water supply from water source $i$ during period $k$ to

313 user $j\left(\right.$ yuan $\left./ \mathrm{m}^{3}\right)$.

314 (3) Cost for reclaimed water supply

$315 f_{3}^{ \pm}=\sum_{j}^{J} \sum_{k}^{K}\left(I R W_{j k}^{ \pm} \times C R I N_{j k}^{ \pm}\right)$ 
where $C R I N_{j k}^{ \pm}$is unit cost of water supply from reused water to user $j$ during

317 planning period $k$ (yuan $\left./ \mathrm{m}^{3}\right)$.

318 (4) Cost for wastewater treatment

$f_{4}^{ \pm}=\sum_{i}^{I} \sum_{j}^{J} \sum_{k}^{K} \sum_{h}^{H}\left(T_{i j k}^{ \pm}+I R W_{j k}^{ \pm}-p_{h}^{l^{*}} \times D_{i j k h}^{ \pm}\right) \times C W W T_{j k}^{ \pm} \times \beta_{j k}^{ \pm}$

where $C W W T_{j k}^{ \pm}$is unit cost of wastewater treatment for user $j$ during period $k$

$\left(\mathrm{yuan} / \mathrm{m}^{3}\right) ; \beta_{j k}^{ \pm}$is the discharge coefficient of wastewater per unit consumption of water during period $k$ for user $j$.

Therefore, the objective function of ITSPF-IPWRM model for industrial park water resource management could be calculated as follows:

$\max f^{ \pm}=\frac{\text { System net benefit }}{\text { Total water consumption }}$

$$
=\frac{f_{1}^{ \pm}-f_{2}^{ \pm}-f_{3}^{ \pm}-f_{4}^{ \pm}}{\sum_{i=1}^{I} \sum_{j=1}^{J} \sum_{k=1}^{K} \sum_{h=1}^{H}\left(T_{i j k}^{ \pm}+I R W_{j k}^{ \pm}-D_{i j k h}^{l \pm}\right)}
$$

Then equations (6.6) to (6.13) present the constraints of ITSPF-IPWRM model:

a) Water supply constraints

The total amount of water allocation to different plants should be lower than the supplying capacity of each water sources:

$$
q_{i h}^{ \pm} \geq \sum_{j=1}^{J}\left(T_{i j k}^{ \pm}-D_{i j k h}^{l \pm}\right) \quad \forall j, k, h
$$

331 where $q_{i h}^{ \pm}$is total water available for source $i$ under water inflow level $h$ $332\left(\mathrm{~m}^{3} /\right.$ day $)$.

b) The amount of water consumption for different water user plants

334 The total water supply to the water user plants should be larger than the lowest water consumption: 
$336 \quad \sum_{i=1}^{I}\left(T_{i j k}^{ \pm}-D_{i j k h}^{l \pm}\right)+I R W_{j k}^{ \pm} \geq I N D W_{j k}^{ \pm} \quad \forall j, k, h$

337 where $I N D W_{j k}^{ \pm}$is the amount of water demand for user $j$ during period $k$ $338 \quad\left(\mathrm{~m}^{3} /\right.$ day $)$.

339 c) Wastewater treatment capacity

$$
\sum_{i}^{I} \sum_{j}^{J}\left(T_{i j k}^{ \pm}-D_{i j k h}^{l \pm}+I R W_{j k}^{ \pm}\right) \times \beta_{j k}^{ \pm} \leq S W T_{k}^{ \pm} \quad \forall j, k, h
$$

341 where $S W T_{k}^{ \pm}$is the capacity for sewage treatment during period $k \quad\left(\mathrm{~m}^{3} /\right.$ day).

342 d) Water supply capacity of waterworks

343 The water allocation should be lower than the maximum water supply ability of

344 waterworks:

$345 \quad \sum_{i}^{I} \sum_{j}^{J}\left(T_{i j k}^{ \pm}-D_{i j k h}^{l \pm}\right) \leq T W C_{k}^{ \pm} \quad \forall j, k, h$

346 where $T W C_{k}^{ \pm}$is the capacity for water treatment plant during period $k$ ( $\mathrm{m}^{3} /$ day).

e) Water reuses capacity

348 The wastewater after been treated can only be reused by themselves due to the

349 limitation of reusing technology and water quality. For each water using plant, the 350 recycled water should be lower than the capacity and the maximum treatment ability 351 of of reused water:

$$
I R W_{j k}^{ \pm} \leq\left[\sum_{i=1}^{I}\left(T_{i j k}^{ \pm}-D_{i j k h}^{I \pm}\right)+I R W_{j k}^{ \pm}\right] \times \theta_{j k}^{ \pm} \leq I R W T_{j k}^{ \pm} \quad \forall j, k, h
$$

353 where $I R W T_{j k}^{ \pm}$is the capacity for water recycling treatment to user $j$ during period $354 k\left(\mathrm{~m}^{3} /\right.$ day $) ; \quad \theta_{j k}^{ \pm}$is the recycling rate of water consumption during period $k$ for user $355 j$. 
$357 \quad \sum_{h=1}^{H} p_{h}^{l^{*}}=1$

$358 \quad H P \leq b$

359 where $P=\left(p_{1}, \mathrm{~L}, p_{N}^{t}\right) \in R^{N},(h=1 \mathrm{~L} N) ; H=\left(h_{i j}\right)$ and $\quad b=\left(b_{i}\right)$ are $s \times N$ and

$360 s \times l$ fixed matrices, respectively.

361 g) Technical constraint

$T \max _{i j k}^{ \pm} \geq T_{i j k}^{ \pm} \geq D_{i j k h}^{l \pm} \geq 0 \quad \forall i, j, k, h$

363 where $T \max _{i j k}^{ \pm}$is maximum allowable allocation amount from source $i$ to user $j$

364 in period $k\left(\mathrm{~m}^{3} /\right.$ day $)$.

3654 Application

$366 \quad 4.1$ Statement of problems

367 Electric power, electronic communication, machinery, food, beverage and 368 pharmaceutical factories are considered the top six major water user plants in 369 industrial park (The People's Government of Tianjin Binhai New Area, 2016). 370 Assuming the planning horizon is fifteen-years and divide into three periods (five 371 years each) to demonstrate the dynamic characteristics in the industrial park water 372 system. Since the reclaimed water can satisfy the low quality of water demand 373 processes, consider the wastewater of factories can be reused by themselves after 374 simple treatment which can reduce the consumption of high quality water. Therefore, 375 water will be supplied from the two sources (e.g., surface water and underground water) to each plant with different amount and operational cost. And the reclaimed water will not be considered in pharmaceutical factory because of the security of production and public acceptance barriers.

379 In this research, interval parameters are applied to solve imprecise uncertainties, 380 which are related to many constraints such as water demands, the costs of water 
supply, the capacities of wastewater treatment and reclaimed water supply, the availability of water resources and so on. The research data are sourced from comprehensive review of statistical yearbooks, governmental reports and many other documents. According to the current situation of industrial park water consumption, Table 1 lists the target water demand for six plants under different water sources. Table 2 presents the economic and operational parameters to each water user plant within industrial park. The industrial park development will obtain benefits, when it meets the need of water using plant. If the promised amount of water supply is not satisfy, it will cause a reduced net system benefit. Under the circumstances, water using plants will encounter higher water priced and production must be reduced to curtail its water demand (Huang and Loucks, 2000). In this research, surface water and groundwater are divided into low, medium and high water inflow levels due to its randomness and variability among different years. Table 3 lists the availability of surface water and underground water and their corresponding probabilities of stream inflow levels. And ITSPF-IPWRM will obtain the lower and upper bound of the objective function at extreme points as the probabilities of the stream inflow fluctuated within their intervals (Kofler, 2001; Fan et al., 2012b) (Table 4, i.e., S1, S2, S3 and S4).

Table 1 Water allocation targets for each plant from different water source (unit: $\mathrm{m}^{3} /$ day)

\begin{tabular}{llllllll}
\hline $\begin{array}{l}\text { Planning } \\
\text { period }\end{array}$ & Water & Plant & & & & & \\
& source & $j=1$ & $j=2$ & $j=3$ & $j=4$ & $j=5$ & $j=6$ \\
\hline$k=1$ & $i=1$ & {$[2680,3580]$} & {$[1670,2540]$} & {$[1768,2458]$} & {$[1330,1870]$} & {$[1145,1921]$} & {$[1453,2363]$} \\
& $i=2$ & {$[840,1730]$} & {$[865,1215]$} & {$[320,525]$} & {$[1150,2010]$} & {$[1370,2230]$} & {$[550,1200]$} \\
$k=2$ & $i=1$ & {$[3350,4260]$} & {$[2447,3137]$} & {$[2063,2793]$} & {$[2350,3040]$} & {$[2619,3449]$} & {$[2116,3096]$} \\
& $i=2$ & {$[910,1820]$} & {$[730,1130]$} & {$[280,610]$} & {$[1359,2289]$} & {$[1860,2790]$} & {$[720,1570]$} \\
$k=3$ & $i=1$ & {$[4670,5700]$} & {$[3136,4046]$} & {$[2936,3816]$} & {$[3319,4089]$} & {$[3015,3995]$} & {$[3119,3989]$} \\
& $i=2$ & {$[1070,2050]$} & {$[910,1510]$} & {$[540,940]$} & {$[1623,2273]$} & {$[2030,2680]$} & {$[940,1290]$} \\
\hline
\end{tabular}


$400{ }^{1}(i=1$ is surface water, $i=2$ is underground water; $j=1$ is electric power factory; $j=2$ is electronic 401 communication factory; $j=3$ is machinery factory; $j=4$ is food factory; $j=5$ is beverage factory; $j=6$ is 402 medicine factory. And $i, j$ represent the same in context.)

403 Table 2 Economic and operational parameters used in the optimization model

\begin{tabular}{|c|c|c|c|c|c|c|}
\hline Planning period & $j=1$ & $j=2$ & $j=3$ & $j=4$ & $j=5$ & $j=6$ \\
\hline \multicolumn{7}{|c|}{ Net benefit when water demand is satisfied (yuan $/ \mathrm{m}^{3}$ ) } \\
\hline$k=1$ & {$[335,390]$} & {$[285,340]$} & {$[275,330]$} & {$[265,320]$} & {$[250,305]$} & {$[225,280]$} \\
\hline$k=2$ & {$[380,435]$} & {$[310,365]$} & {$[300,355]$} & {$[280,335]$} & {$[270,325]$} & {$[255,310]$} \\
\hline$k=3$ & {$[435,490]$} & {$[350,405]$} & {$[330,385]$} & {$[310,365]$} & {$[300,355]$} & {$[285,340]$} \\
\hline \multicolumn{7}{|c|}{ Penalty when water demand is not delivered (yuan $/ \mathrm{m}^{3}$ ) } \\
\hline$k=1$ & {$[430,485]$} & {$[395,450]$} & {$[375,430]$} & {$[370,425]$} & {$[345,400]$} & {$[340,395]$} \\
\hline$k=2$ & {$[475,530]$} & {$[405,460]$} & {$[390,445]$} & {$[385,440]$} & {$[360,415]$} & {$[355,410]$} \\
\hline$k=3$ & {$[530,585]$} & {$[445,500]$} & {$[420,475]$} & {$[400,455]$} & {$[385,440]$} & {$[370,425]$} \\
\hline \multicolumn{7}{|c|}{ The amount of water demand $\left(\mathrm{m}^{3} / \mathrm{d}\right)$} \\
\hline$k=1$ & {$[3384,5454]$} & {$[1036,1356]$} & {$[913,1033]$} & {$[3745,3845]$} & {$[3120,3210]$} & {$[1935,1985]$} \\
\hline$k=2$ & {$[4884,6084]$} & {$[2236,2306]$} & {$[1113,1903]$} & {$[5545,5745]$} & {$[4620,4820]$} & {$[2100,2150]$} \\
\hline$k=3$ & {$[5284,7484]$} & {$[3036,3336]$} & {$[2713,2803]$} & {$[6045,6245]$} & {$[5320,5520]$} & {$[3500,3550]$} \\
\hline
\end{tabular}

404 Table 3 Description of stream flow distribution $\left(\mathrm{m}^{3} / \mathrm{d}\right)$

\begin{tabular}{|c|c|c|c|}
\hline & Inflow level & & \\
\hline & Low $(h=1)$ & Medium $(h=2)$ & High $(h=3)$ \\
\hline$i=1$ & {$[10990,14090]$} & {$[11270,15770]$} & {$[23445,28645]$} \\
\hline$i=2$ & {$[4143.9,8543.9]$} & {$[4207.8,9107.8]$} & {$[11055.4,13355.4]$} \\
\hline
\end{tabular}

Table 4 Description of partial probability distribution

\begin{tabular}{lllll}
\hline & Scenario 1 & Scenario 2 & Scenario 3 & Scenario 4 \\
\hline Low $(h=1)$ & 0.1 & 0.1 & 0.2 & 0.2 \\
Medium $(h=2)$ & 0.7 & 0.6 & 0.6 & 0.5 \\
High $(h=3)$ & 0.2 & 0.3 & 0.2 & 0.3
\end{tabular}

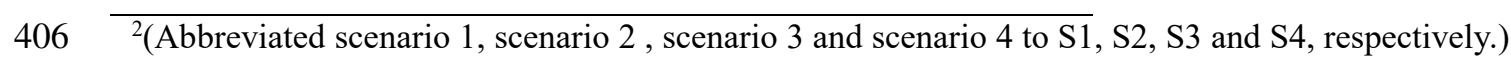

$407 \quad 4.2$ Results and analysis

408 Figs. 4 to 5 depict the amount of utilization and shortage of each water source as the 409 water change from low to high inflow level in each scenario. It can be seen that more 410 water would be allocated into the system as the probability of high water inflow level 
is getting higher under the same probability of low water inflow level. The reason is

412

413 that the system would have more water resources allocated to each enterprise as the probability of high flow level increased. As for S2, for instance, $[2.81,3.84] \times 10^{3}$ $\mathrm{m}^{3} /$ day, $[11.27,14.95] \times 10^{3} \mathrm{~m}^{3} /$ day and $14.95 \times 10^{3} \mathrm{~m}^{3} /$ day of surface water would be allocated in period 2 under low, medium and high water inflow levels, respectively (Fig.4). Compared to S2, it can be seen that the surface water distribution for S1 would be $[43.20,51.00] \%,[0,4.80] \%$ and $[68.40,70.00] \%$ lower than $\mathrm{S} 2$ under low, medium and high water inflow levels, respectively. Moreover, the amount of water shortage for S2 would be lower than S1 over periods under the same water inflow level (Fig.5). For example, the amount of water shortage for S2 under low water inflow level would be $[9.70,17.50] \%,[10.60,13.00] \%$ and $[0.70,4.90] \%$ lower than $\mathrm{S} 1$ in period 1, 2 and 3, respectively. Therefore, as higher probability of high water inflow level, the less amount of water shortage would be. Under the condition of sufficient water resource, the industrial park would be more inclined to use high-quality water to meet the quality of production, and the consumption of reclaimed water would be reduced. As illustrated in Fig.6, the utilization of reclaimed water for $\mathrm{S} 1$ would be $[7.80,8.89] \times 10^{3} \mathrm{~m}^{3} /$ day, $[19.04,20.03] \times 10^{3} \mathrm{~m}^{3} /$ day and $[24.18$, $24.72] \times 10^{3} \mathrm{~m}^{3} /$ day over periods. And there would be $[6.39,7.14] \times 10^{3} \mathrm{~m}^{3} /$ day, $[17.48$, $18.83] \times 10^{3} \mathrm{~m}^{3} /$ day and $[21.60,22.87] \times 10^{3} \mathrm{~m}^{3} /$ day for $\mathrm{S} 2$ in period 1,2 and 3 , respectively. Compared to S2, the risk of water shortage would be higher in S3 due to the higher probability of low water inflow level and thus the water supply of S3 is lower than S2. For example, the deficiency of surface water for S3 would be [5.13, $9.22] \%,[25.00,40.00] \%$ and $[77.33,85.72] \%$ higher than $\mathrm{S} 2$ in period 1 under low, medium and high water inflow levels, respectively. The industrial park would increase the utilization of reclaimed water to meet their own production needs when the risk of 
water shortage increased. In this case, the utilization of reclaimed water for S3 would be $[8.03,8.91] \times 10^{3} \mathrm{~m}^{3} /$ day, $[19.96,20.36] \times 10^{3} \mathrm{~m}^{3} /$ day and $[24.18,24.87] \times 10^{3} \mathrm{~m}^{3} /$ day, which higher than S1 and S2. At last, the allocation of surface water for S4 would be higher than S3 due to the probability of high water inflow level increased from 0.2 to 0.3. In general, the total underground water supply is lower than surface water. As for S2 in period 2, for instance, the allocation of underground water would be [25.50, $43.70] \%,[60.80,62.70] \%$ and $60.80 \%$ lower than surface water under low, medium and high water inflow levels, respectively. Therefore, surface water would be the major supply source in industrial park. Following surface water, the trend of underground water would be allocated in a similar way under the change of water inflow level. For example, the allocation of underground water for S2 would be $[26.68,29.12] \%,[1.96,2.38] \%$ and $[39.89,67.81] \%$ higher than $\mathrm{S} 1$ in period 1 under low, medium and high water inflow levels, respectively. And the amount of water shortage would be $[29.62,29.36] \%,[0,68.18] \%$ and $[58.62,63.24] \%$ lower than $\mathrm{S} 1$.

To sum up, the amount of water supply would increase with the probability of high water inflow level when the probability of low inflow level is equal. In order to meet the quality of production, the industrial park is more inclined to high quality water and the water distribution for surface water and underground water would increase, while the amount of reclaimed water would decrease. When the probability of low water inflow level increased, the risk of water shortage would be higher and the industrial park would increase the utilization of reclaimed water to satisfy its own production needs. Surface water is the main water supply for the industrial park, followed by underground water. Under the higher risk of water shortage, reclaimed water could replace the high quality water to satisfy the process of low quality water use and reduce low benefit caused by high water shortage. This could avoid the 
463
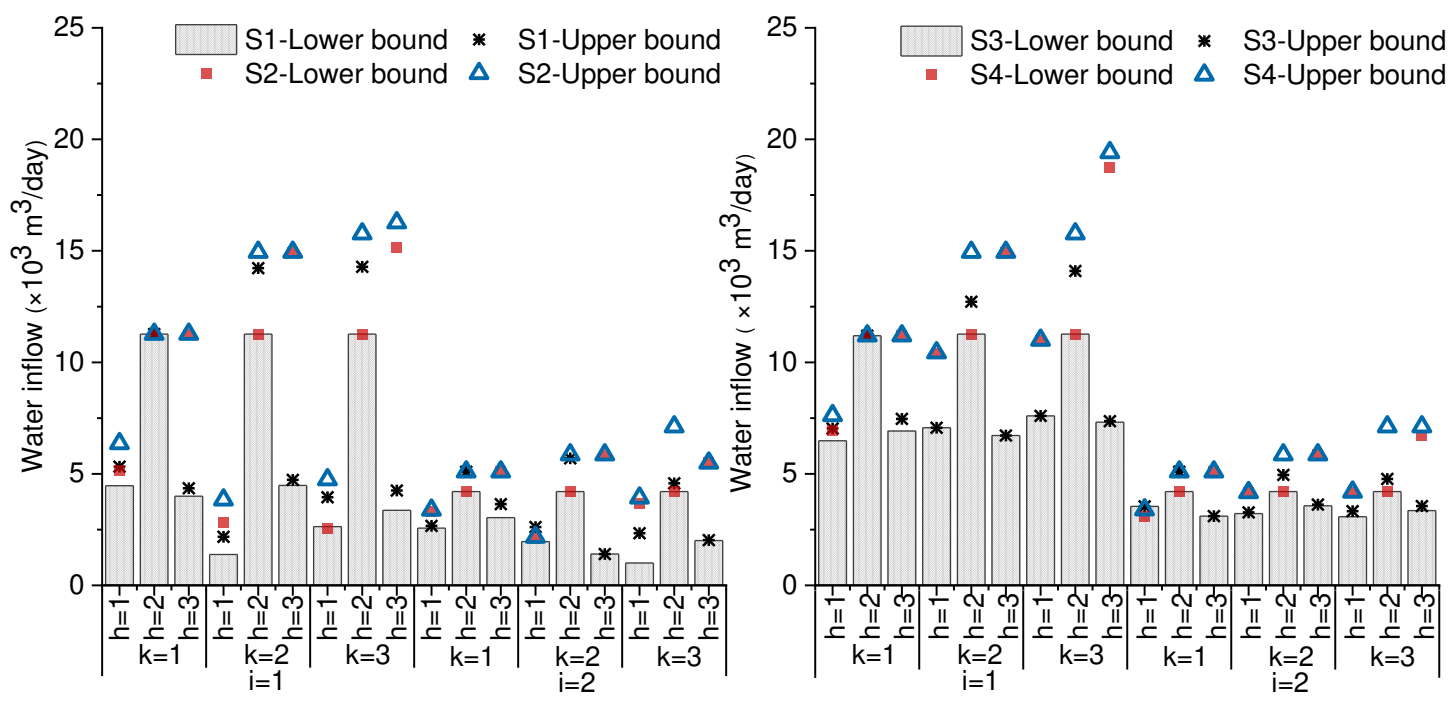

Fig.4 Optimal allocation schemes for different water sources in each scenario over periods $(k=1$ is period $1 ; k=2$ is period $2 ; k=3$ is period $3 ; h=1$ is low inflow level; $h=2$ is medium inflow level; $h=3$ is high inflow level)
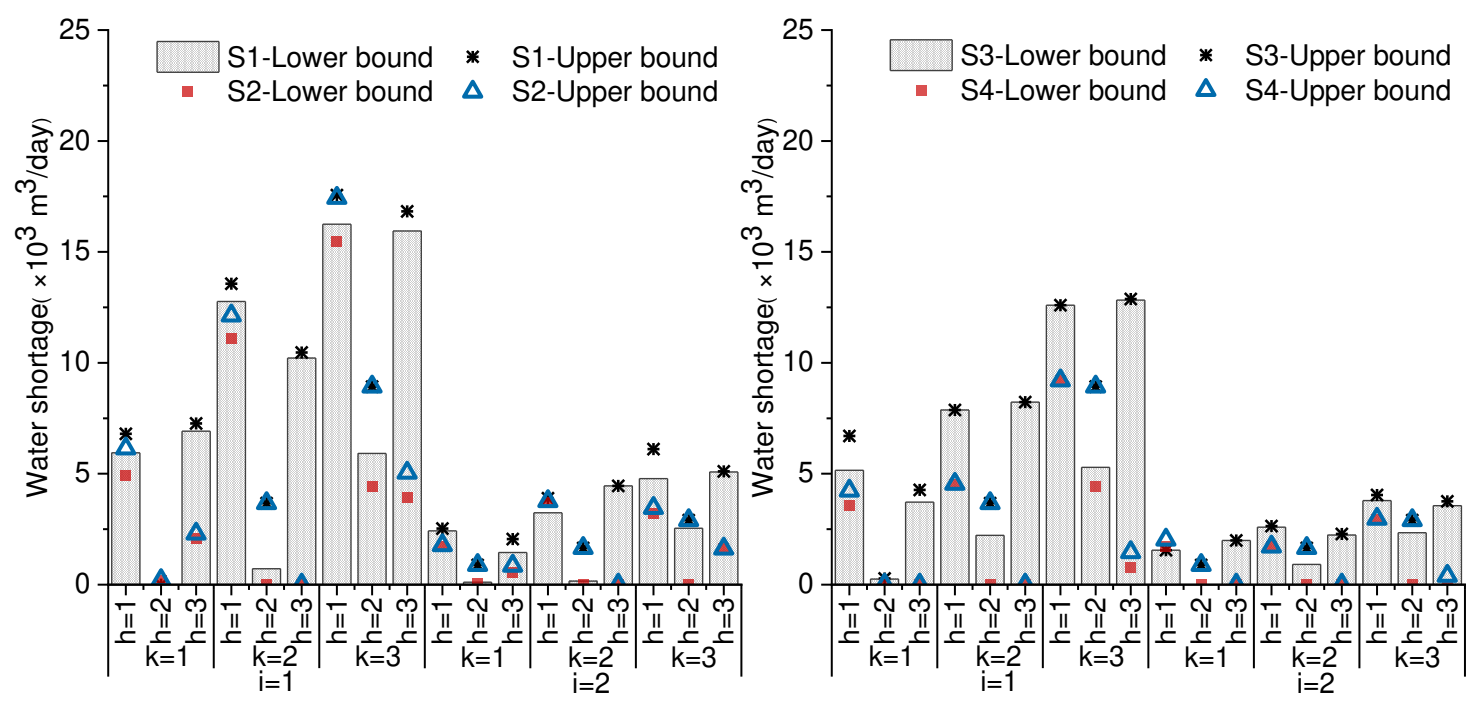

Fig.5 The amount of water shortage in each scenario over periods 
469

470

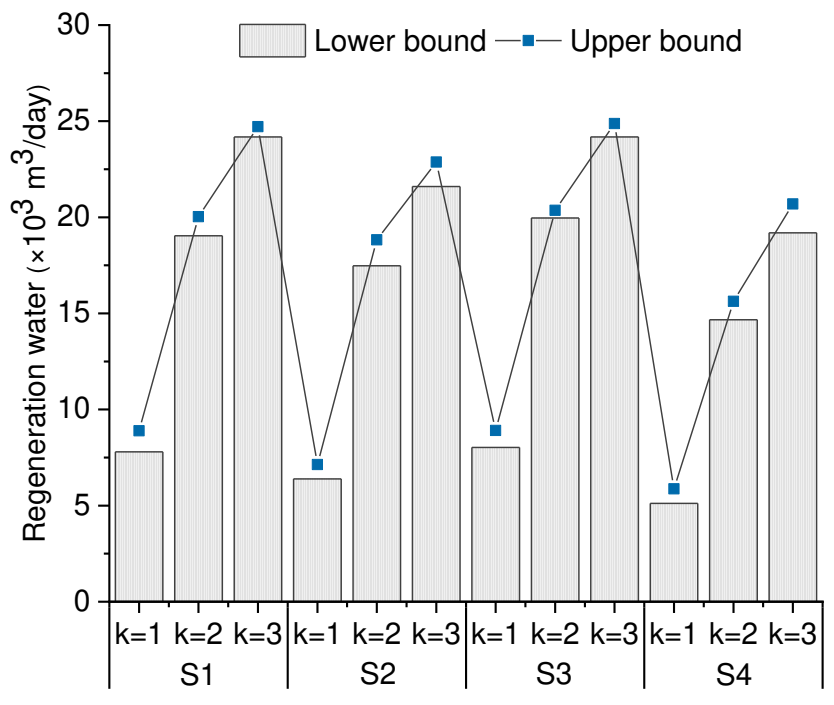

Fig.6 The amount of reclaimed water in each scenario over periods

In order to clarify the amount of water allocation and shortage of each plant in different periods, the $\mathrm{S} 1$ under low water inflow level is taken as an example due to the water consumption for each plant in different scenario is similar. Fig. 7 presents the changes of water allocation and shortage in different periods for S1 under the low water inflow level. The water shortage of each plant would gradually increase over periods under the same water inflow level when surface water is used as the water supply source. For example, the amount of water shortage for electric power would be $[2.49,3.30] \times 10^{3} \mathrm{~m}^{3} /$ day in period 1 under low water inflow level, which would be $[1.5,9.8] \%$ and $[26.9,29.4] \%$ lower than period 2 and 3 . And the allocation of surface water would be $[0,0.81] \times 10^{3} \mathrm{~m}^{3} /$ day, $[0,0.59] \times 10^{3} \mathrm{~m}^{3} /$ day and $[0,1.26] \times 10^{3} \mathrm{~m}^{3} /$ day in period 1, 2 and 3, respectively. This is attribute to the amount of water available can not meet the increasing demand of electric power, resulting in a gradual increase in water shortage. Besides, the amount of water shortage for electric power would be the highest compared to other plants. For example, the deficiency of surface water for electronic communication, machinery and medicine factory would be $1.70 \times 10^{3}$ $\mathrm{m}^{3} /$ day, $1.80 \times 10^{3} \mathrm{~m}^{3} /$ day and $[16.31,66.31] \mathrm{m}^{3} /$ day, respectively. And the amount of water shortage for food and beverage factory would be the lowest which the amount 
of water allocation would reach the upper bound of its water demand and would be $1.33 \times 10^{3} \mathrm{~m}^{3} /$ day and $1.75 \times 10^{3} \mathrm{~m}^{3} /$ day. The reason is that food and beverage factory would be the second and third largest water demand in the industrial park. And compared to electric power, the largest water-consuming factory in the park, the sewage discharge of food and beverage factory would be low. Therefore, the water demand of food and beverage factory would be preferentially satisfied. Reclaimed water would be used to replace high quality water (i.e., surface water and underground water) to meet the low water quality requirements in some processes of factory, when factories challenged by water shortage. Fig. 8 illustrated the utilization of reclaimed water of each factory in S1. The amount of reclaimed water would gradually increased in period 1,2 and 3. For example, the utilization of reclaimed water for electric power would be $[4.08,4.65] \times 10^{3} \mathrm{~m}^{3} /$ day, $4.88 \times 10^{3} \mathrm{~m}^{3} /$ day and $5.28 \times 10^{3} \mathrm{~m}^{3} /$ day over periods. For period 2 and 3 , there would be $[5.10,19.58] \%$ and $[13.71,29.37] \%$ higher than period 1. Compared to other factories, electric power would have the largest utilization of reclaimed water. For example, the amount of reclaimed water for electric power, electronic communication, machinery, food and beverage factory would be $[4.08,4.65] \times 10^{3} \mathrm{~m}^{3} /$ day, $[1.04,1.36] \times 10^{3} \mathrm{~m}^{3} /$ day, $[0.91$, $1.03] \times 10^{3} \mathrm{~m}^{3} /$ day, $1.76 \times 10^{3} \mathrm{~m}^{3} /$ day and $[0,0.09] \times 10^{3} \mathrm{~m}^{3} /$ day in $\mathrm{S} 1$ during period 1 . In conclusion, the deficiency of water for each factory would gradually increase over periods under the same water inflow level due to the water availability can not satisfy the increasing water demand of factories. In order to satisfy their own water demand, they would increase the utilization of reclaimed water to replace the high quality water to satisfy the process of low quality water use . 


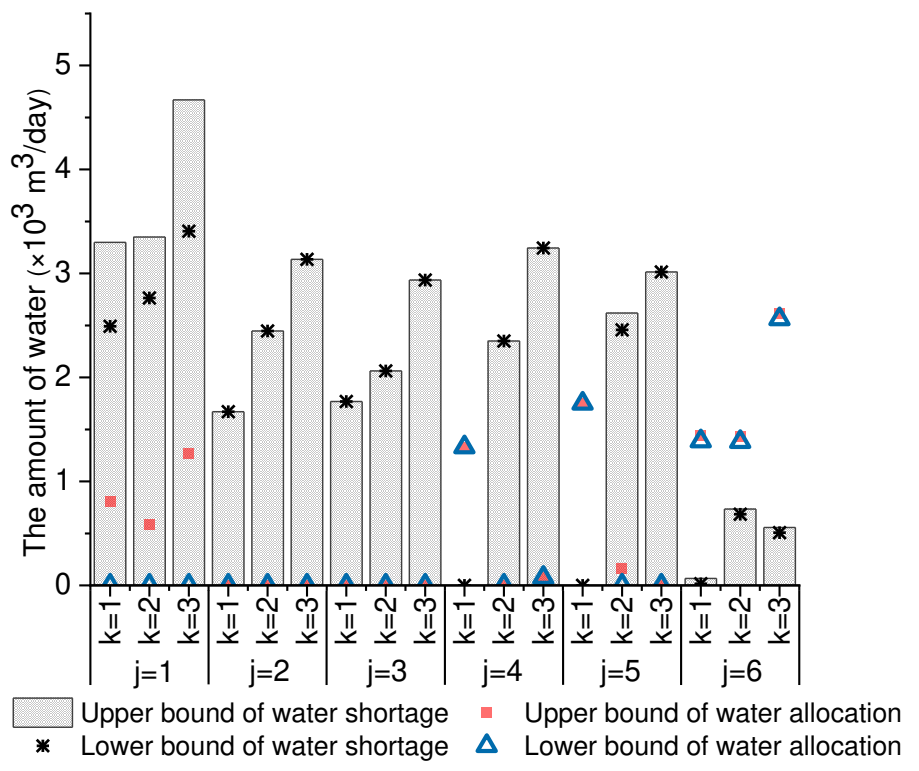

512 Fig.7 The amount of water allocation and shortage for the six water user plants under low water inflow

513 level in S1 during the planning horizon

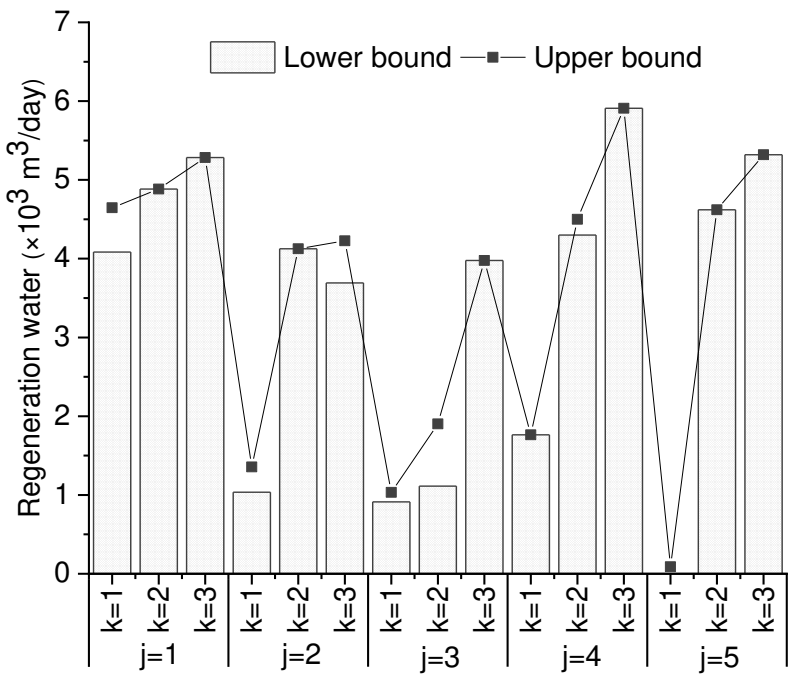

515 Fig.8 The amount of reclaimed water for the six water user plants in S1 during the planning horizon

Fig.9 present the upper and lower bounds of the modeling objective function, for instance, net system benefits and recourse cost. In general, electric power factory has the highest net benefit over all planning periods and water inflows scenarios. For S1 during period 1 , the total net benefit for electric power factory is $[4.10,5.20] \times 10^{6}$ yuan, while electronic communication, machinery and medicine factory is $[1.30,1.80]$ $\times 10^{6},[1.10,1.30] \times 10^{6}$ and $[4.50,5.60] \times 10^{5}$ yuan, respectively. Meanwhile, the net 

power in S2 from period 1 to 3, respectively. The more water allocates, the higher net

525 benefit factory can get. Besides, the factories would have higher recourse cost under 526 higher benefit. The recourse cost for electric power, electronic communication, 527 machinery and medicine factory during period 1 in $\mathrm{S} 1$ would be $[4.30,6.00] \times 10^{5}$, $[3.00,3.40] \times 10^{5},[2.40,3.70] \times 10^{5}$ and $[0.18,2.70] \times 10^{4}$ yuan, respectively. It can

529 be seen that electric power factory should be ensured because it provide the highest 530 benefit $\left(\mathrm{NB}_{j k}^{ \pm}\right)$when its water demand is met, whereas it is subject to the highest 531 penalty if the promised water is not delivered. In comparison with that, medicine 532 factory would correspond to lower benefit and penalties. Consequently, the allocation water to medicine factory should be diminished firstly in the case of inadequate water 534 supplies.
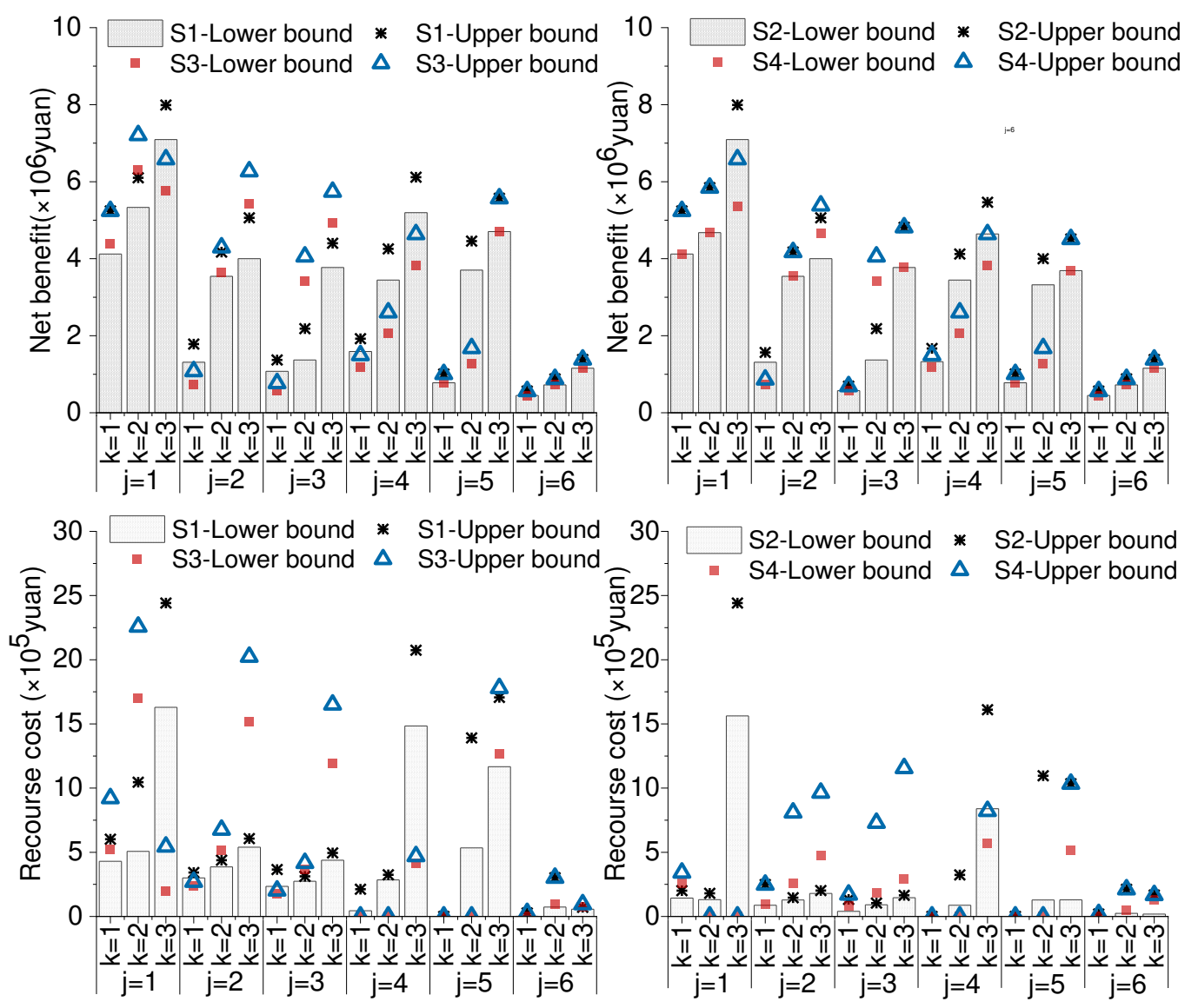
Fig.9 Net system benefit and recourse cost for each factory during the three planning periods

Generally, the above results present different water allocation schemes under different scenarios, and different economic efficiency of water use would obtain (Fig.10). In detail, the objective function value would be [98.10, 126.80], [98.10, 124.10], [95.00, 122.20] and $[95.70,120.60]$ under S1, S2, S3 and S4, respectively. The objective function values gradually decrease as a result of higher probabilities for low inflow and lower probabilities of medium inflow. As the probabilities of low inflow levels for $\mathrm{S} 1, \mathrm{~S} 2, \mathrm{~S} 3$ and S4 would be $0.1,0.1,0.2$ and 0.2 , respectively; while the probabilities of medium inflow levels for S1, S2, S3 and S4 would be 0.7, 0.6, 0.6 and 0.5, respectively. Therefore, more water resources in S1 would be available. Above all, the final optimized value for the objective function would be $f_{\text {opt }}^{ \pm}=\left[\min \left(f^{1-}, f^{2-}, f^{3-}, f^{4-}\right), \max \left(f^{1+}, f^{2+}, f^{3+}, f^{4+}\right)\right]=[95.00,126.80]$. It presents the range for the objective function over the planning horizon.
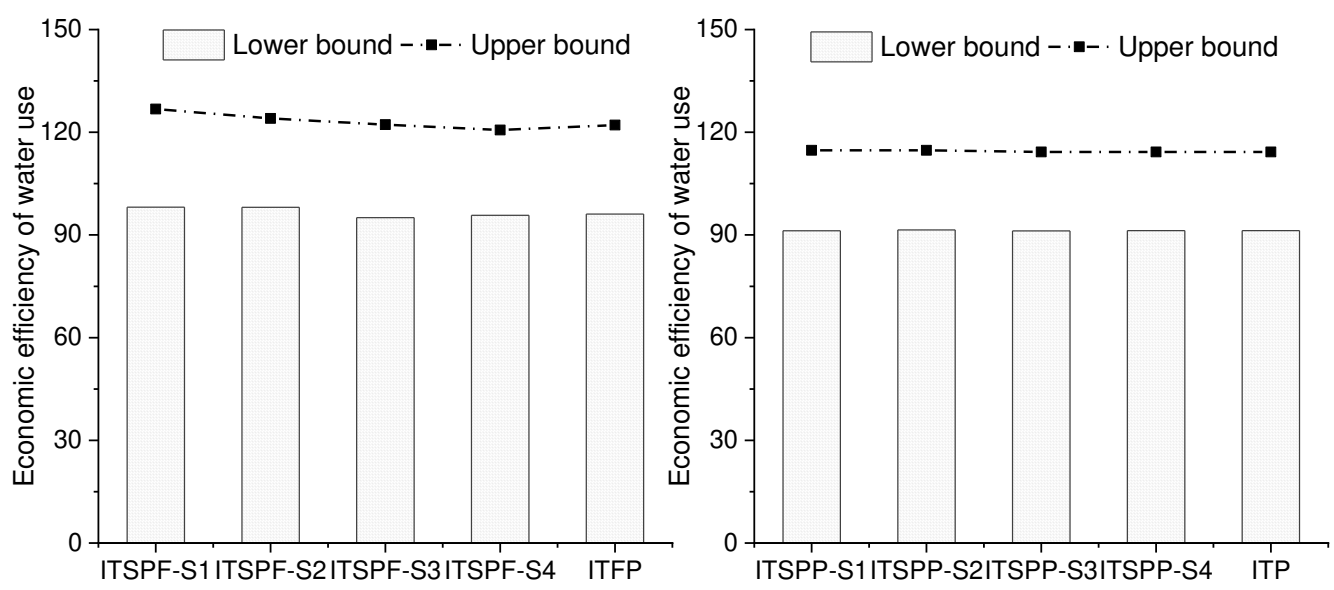

Fig.10 The economic efficiency of water use for ITSPF, ITFP, ITSPP and ITP model

\subsection{Discussion}

In this research, the proposed ITSPF-IPWRM method can obtain optimal efficiency between system benefit and water allocation in industrial park. The interval solutions present the dynamics of water allocation patterns which are sensitive to uncertain inputs. Multiple models are introduced for comparison to confirm the effectiveness 
and reliability of ITSPF model. The proposed inexact two-stage fractional

557 programming (ITFP) can tackle interval uncertainty except uncertain probability.

558 Hence, an ITFP model is generated to address industrial park water resources

559 management problem by introducing the mid-values to represent the interval

560 probabilities. Moreover, the ITSPF model will convert to inexact two-stage stochastic

561 partial programming (ITSPP) as the planners pay more attention to maximize system

562 benefits. And the inexact two-stage programming (ITP) with deterministic probability

563 distribution is also considered. Therefore, three optimization models that originated

564 from the ITSPF model are established and listed in Table 5 and the optimal values of

565 different optimization models are shown in Fig.11.

566 Table 5 Optimization models for comparing with ITSPF model

\begin{tabular}{lll}
\hline Model & Objective function & Constraints \\
\hline ITFP & Maximize the economic efficiency of water use Same as the ITSPF \\
& deterministic probability distribution \\
ITSPP & Maximize the system economic benefit with partial \\
& probability distribution \\
ITP & Maximize the system economic benefit with \\
& deterministic probability distribution
\end{tabular}



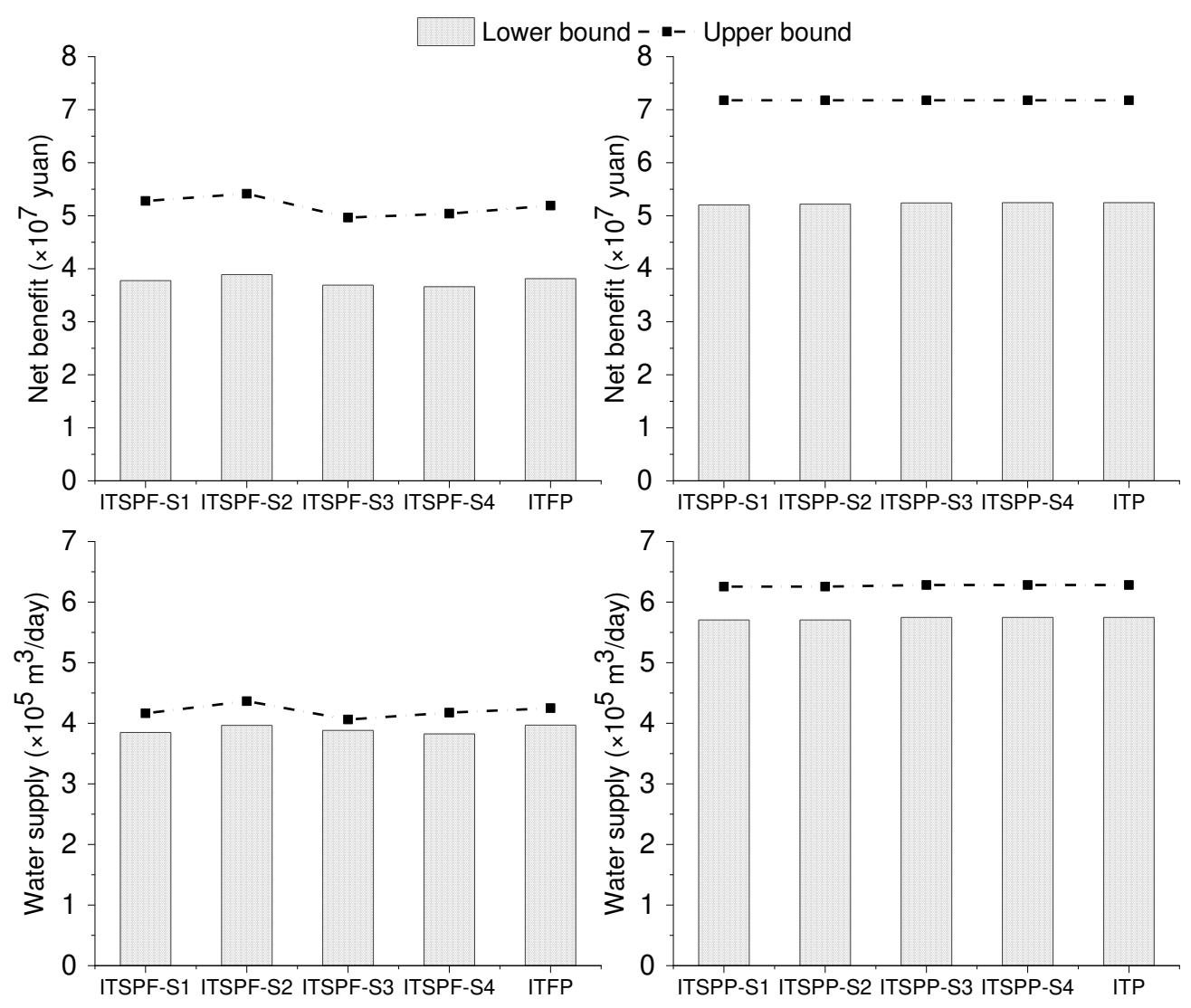

Fig.11 Net benefit and water supply formulate by ITSPF, ITFP, ITSPP and ITP model

In terms of the system economic benefit as shown in Fig.11, the solution for ITSPF would be $[3.66,5.41] \times 10^{7}$ yuan, while ITFP model would be $[3.81,5,19] \times 10^{7}$ yuan.

The range of system economic benefit for ITSPP would be $[5.21,7.18] \times 10^{7}$ yuan

when consider the probability distribution partially. And $[5.25,7.18] \times 10^{7}$ yuan would be obtained formulating by ITP model. It can be seen that the values without considering partial probability distribution (i.e., ITFP and ITP model) are obviously located within ITSPF and ITSPP, respectively. As for water supply, there would be $[3.85,4.17] \times 10^{5} \mathrm{~m}^{3} /$ day for ITSPF model in S1. And the amount of water supply would be increased to $[3.97,4.36] \times 10^{5} \mathrm{~m}^{3} /$ day as the probability of high stream inflow ascend from 0.2 to 0.3 . The value would go down to $[3.88,4.06] \times 10^{5} \mathrm{~m}^{3} /$ day since the probability of low stream inflow would be higher. And the amount of water allocation would be $[3.83,4.18] \times 10^{5} \mathrm{~m}^{3} /$ day in $\mathrm{S} 4$. Therefore, the final optimized 
range for ITSPF model would be $[3.83,4.36] \times 10^{5} \mathrm{~m}^{3} /$ day. For ITFP model, the final

582 amount of water allocation would be $[3.97,4.25] \times 10^{5} \mathrm{~m}^{3} /$ day, which is obviously located within ITSPF model. Moreover, the optimal water allocation would be [5.71, $6.26] \times 10^{5} \mathrm{~m}^{3} /$ day, $[5.71,6.26] \times 10^{5} \mathrm{~m}^{3} /$ day, $[5.75,6.28] \times 10^{5} \mathrm{~m}^{3} /$ day and $[5.75$, $6.28] \times 10^{5} \mathrm{~m}^{3} /$ day for ITSPP model in $\mathrm{S} 1, \mathrm{~S} 2, \mathrm{~S} 3$ and $\mathrm{S} 4$, respectively. It can be seen that the water allocation would be the same under the same probability of low stream inflow formulating by ITSPP mode. Therefore, the ITSPF results can provide wider adjustable ranges than ITSPP, thus ITSPF can offer more practical possibilities for obtaining satisfactory alternatives under various system conditions without missing possible solution set.

As depicted in Fig.10, the economic efficiency of water use for ITFP model would be $[96.10,122.10]$. Although the ITFP method can generate feasible interval solution for practical problems with deterministic probability distribution, it would ignore many other feasible alternatives as the stochastic partial information is varied within their spectra and can not acquire the final fluctuating interval of the objective function when the parameters and decision variables fluctuate within their intervals. For ITSPP model, the objective function value would be [91.22, 114.74], [91.48, 114.74], [91.17, 114.24] and [91.27, 114.24] under S1, S2, S3 and S4, respectively. Therefore, the final optimized value for the objective function would be [91.17, 114. 74]. And the optimal value for ITP model would be $[91.25,114.24]$. Although the systems would have higher system economic benefit with mono-objective, it would consume a higher amount of water and present lower levels of system efficiency. For example, the system economic benefit for ITSPP and ITP is $[32.56,42.13] \%$ and $[32.55,43.26] \%$ higher than ITSPF, respectively. But their system efficiency would be $[4.03,9.51] \%$ and $[3.95,9.90] \%$ lower than ITSPF. 
In comparison, the ITSPF model can address uncertain probability through generating interval solutions under corresponding extreme points effectively. Uncertain cost coefficients, resource availability, and constraint parameters can be totally addressed in ITSPF. The ITSPF model can be transformed into an ITFP model and then obtained decision intervals under each extreme point. On one hand, ITSPF advances conventional FP by tackling probability distribution of water inflow expressed as interval and also avoiding the loss of uncertain information during data input. Meanwhile, ITSPF can obtain interval sets that offer plenty of decision alternatives in response to uncertain inputs and wider adjustable ranges can be obtained which can provide a great deal of real-world possibilities for obtaining satisfactory alternatives under multiple system conditions. On the other hand, ITSPF is superior to existing industrial water optimization methods which can simultaneously reflect the economic efficiency of water use while tackling various uncertainties exist in system.

The ITSPF method firstly address the compound uncertainties expressed as the integration of interval numbers, probability distributions and linear partial information under the frame of fractional programming simultaneously. However, the ITSPF method can not deal with fuzzy uncertainty, chance-constrained programming and many other complicated uncertainties in practical environmental management problems. Moreover, the ITSPF approach can not address other type of stochastic information existing in general stochastic partial information. Hence, further studies can concentrate on improving the proposed ITSPF approach to reflect more uncertainties such as integrating with fuzzy flexible programming, chance constrained programming, robust programming, etc. In addition, the proposed ITSPF approach method is not limited to industrial park water resource planning, which is also useful to address other types of resources management issues. 


\section{Conclusions}

632 Inherent uncertainties of solving industrial park water resource management problems will cause great difficulties while considering conflict objectives of system. In this research, an inexact two-stage stochastic partial fractional programming has been established for industrial park water resource management to address dual uncertainties. The contribution of this research can be summarized as follows. Initially, dual uncertainties and ratio problems can be effectively reflected through the ITSPF model, which advance the existing optimization methods through integrating ITSPP model with FP. Additionally, the FP concept has been firstly applied to industrial park water resource management which advance in balancing conflict objectives by considering economic efficiency of water use and reflecting complicated interactions among various system factors. Eventually, it facilitates dynamic analysis for decisions of water allocation within a multi-source, multi-period and multiple uncertain water inflow levels context. The ITSPF model is transformed into two sub-models which reflect the dynamics and interactions between complicated uncertainties and decision processes under various representative scenarios. Therefore, ITSPF can permit in-depth insights of different stream inflow scenarios when the promised water-allocation targets are violated.

The ITSPF method has been applied to industrial park water resources management, 650 which the uncertain probability of stream inflow is tackled by introducing linear 651 partial information. The results indicated that ITSPF-IPWRM can help to identify 652 desired water allocation schemes under compound complexities. Firstly, more high 653 quality water would be allocated into the system and the amount of reclaimed water 654 would be decreased as the probability of high stream inflow level is getting higher 655 while the probability of low stream inflow level is equal. Since the probability of low 
water inflow level is getting higher, the system would gain the higher risk of water shortage and the reclaimed water would be increased to meet the needs of water user plants. Secondly, reclaimed water would be chosen when the water demand of enterprises is getting higher and higher, which would be ascribed to its lower water allocation cost by the companies. Thirdly, the plant with highest benefit $\left(N B_{j k}^{ \pm}\right)$should be ensured because it would gain the highest penalty if the promised water is not delivered. A comparative least-cost water allocation model and model without stochastic partial information have been considered. For one thing, the ITSPF generates feasible alternatives as the stochastic partial information, operational parameters and decision variables are varied within their spectra. For another, it can consume a lower amount of water and present higher levels of system efficiency. Particularly, more uncertainties such as fuzziness can be considered in the future studies. And ITSPF can be exploited in other resources management problems which is helpful for inexact analysis and relative comparisons of two magnitudes.

\section{Reference}

Aviso K B, Tan R R, Culaba A B (2010) Designing eco-industrial water exchange networks using fuzzy mathematical programming. Clean Technol Environ Policy 12:353-363. https://doi.org/10.1007/s10098-009-0252-1.

Bauer S, Linke H J, Wagner M (2020) Optimizing water-reuse and increasing water-saving potentials by linking treated industrial and municipal wastewater for a sustainable urban development. Water Sci Technol 81:1927-1940. https://doi.org/10.2166/wst.2020.257.

Bi R S, Chen C, Tang J, Jia X P, Xiang S G (2019) Two-level optimization model for water consumption based on water prices in eco-industrial parks. Resour Conserv Recycl 146:308-315. https://doi.org/10.1016/j.resconrec.2019.04.004.

Boix M, Montastruc L, Azzaro-Pantel C, Domenech S (2015) Optimization methods applied to the design of eco-industrial parks: a literature review. J Clean Prod 87:303-317. https://doi.org/10.1016/j.jclepro.2014.09.032. 
Boix M, Montastruc L, Pibouleau L, Azzaro-Pantel C, Domenech S (2011) A multiobjective optimization framework for multicontaminant industrial water network design. J Environ Manage 92:1802-1808. https://doi.org/10.1016/j.jenvman.2011.02.016.

Boix M, Montastruc L, Pibouleau L, Azzaro-Pantel C, Domenech S (2012) Industrial water management by multiobjective optimization: from individual to collective solution through eco-industrial parks. J Clean Prod 22:85-97. https://doi.org/10.1016/j.jclepro.2011.09.011.

Boretti A, Rosa L (2019) Reassessing the projections of the world water development report. NPJ Clean Water 2:1-6. https://doi.org/10.1038/s41545-019-0039-9.

Boysen B, Cristobal J, Hilbig J, Guldemund A, Schebek L, Rudolph K U (2020) Economic and environmental assessment of water reuse in industrial parks: case study based on a model industrial park. J Water Reuse Desalin 10: 475-489. https://doi.org/10.2166/wrd.2020.034.

Burek P, Satoh Y, Fischer G, Kahil M, Scherzer A, Tramberend S, Nava L, Wada Y, Eisner S, Flörk M, Hanasaki N, Magnuszewski P, Cosgrove B, Wiberg D (2016) Water futures and solution - fast track initiative (final report). International Institute for Applied Systems Analysis (IIASA).

Charnes A, Cooper W W (1963) Programming with linear fractional functionals. Nav Res Logist Q 9:181-186. https://doi.org/10.1002/nav.3800100123.

Chen H G, Wang Z J (2021) An inexact multi-stage interval-parameter partial information programming model for water resources management under uncertainties. Water Supply 21:2139-2157. https://doi.org/10.2166/ws.2020.265.

Chew I M L, Tan R R, Foo D C Y, Chiu A S F (2009) Game theory approach to the analysis of inter-plant water integration in an eco-industrial park. J Clean Prod 17:1611-1619. https://doi.org/10.1016/j.jclepro.2009.08.005.

Cui L, Li Y P, Huang G H (2015) Planning an agricultural water resources management system: a two-stage stochastic fractional programming model. Sustainability 7:9846-9863. https://doi.org/10.3390/su7089846.

El-Halwagi M M, Gabriel F, Harell D (2003) Rigorous graphical targeting for resource conservation via material recycle/reuse networks. Ind Eng Chem Res 42:4319-4328. https://doi.org/10.1021/ie030318a.

Fan Y R, Huang G H (2012a) A robust two-step method for solving interval linear programming problems within an environmental management context. J Environ Inform 19:1-9. 
https://doi.org/10.3808/jei.201200203.

Fan Y R, Huang G H, Guo P, Yang A L (2012b) Inexact two-stage stochastic partial programming: application to water resources management under uncertainty. Stoch Environ Res Risk Assess 26:281-293. https://doi.org/10.1007/s00477-011-0504-6.

Fasakhodi A A, Nouri S H, Amini M (2010) Water resources sustainability and optimal cropping pattern in farming systems; a multi-objective fractional goal programming approach. Water Resour Manag 24:4639-4657. https://doi.org/10.1007/s11269.010.9683.z.

Hladik M (2010) Generalized linear fractional programming under interval uncertainty. Eur J Oper Res 205:42-46. https://doi.org/10.1016/j.ejor.2010.01.018.

Huang G H, Loucks D P (2000) An inexact two-stage stochastic programming model for water resources management under uncertainty. Civ Eng Environ Syst 17:95-118. https://doi.org/10.1080/02630250008970277.

Ji Y, Ma G, Wei J, Dai Y M (2017) A hybrid approach for uncertain multi-criteria bilevel programs with a supply chain competition application. J Intell Fuzzy Syst 33:2999-3008. https://doi.org/10.3233/JIFS-169351.

Kastner C A, Lau R, Kraft M (2015) Quantitative tools for cultivating symbiosis in industrial parks. Appl Energy 155:599-612. https://doi.org/10.1016/j.apenergy.2015.05.037

Khor C S, Chachuat B, Shah N (2014) Fixed-flowrate total water network synthesis under uncertainty with risk management. J Clean Prod 77:79-93. https://doi.org/10.1016/j.jclepro.2014.01.023.

Kofler E (2001) Linear partial information with applications. Fuzzy Sets Syst 118:167-177. https://doi.org/10.1016/S0165-0114(99)00088-3.

Lawal M, Alwi S R W, Manan Z A, Ho W S (2021) Industrial symbiosis tools-a review. J Clean Prod 280:1-20. https://doi.org/10.1016/j.jclepro.2020.124327.

Li P, Arellano-Garcia H, Wozny G (2008) Chance constrained programming approach to process optimization under uncertainty. Comput Chem Eng 32:25-45. https://doi.org/10.1016/j.compchemeng.2007.05.009.

Liao Z W, Wu J T, Jiang B B, Wang J D, Yang Y R (2007) Design methodology for flexible multiple plant water networks. Ind Eng Chem Res 46:4954-4963. https://doi.org/10.1021/ie061299i.

Lin Q G, Huang G H (2009) Planning of energy system management and GHG-emission control in the municipality of Beijing - an inexact-dynamic stochastic programming model. Energy Policy 
Mani A, Tsai F T C, Paudel K P (2016) Mixed integer linear fractional programming for conjunctive use of surface water and groundwater. J Water Resour Plan Manage-ASCE 142:1-13. https://doi.org/10.1061/(ASCE)WR.1943-5452.0000676.

Marler R T, Arora J S (2010) The weighted sum method for multi-objective optimization: new insights. Struct Multidiscip Optim 41:853-862. https://doi.org/10.1007/s00158-009-0460-7.

Mehra A, Chandra S, Bector C R (2007) Acceptable optimality in linear fractional programming with

Mavrotas G (2009) Effective implementation of the epsilon-constraint method in multi-objective mathematical programming problems. Appl Math Comput 213:455-465. https://doi.org/10.1016/j.amc.2009.03.037.

$$
\text { fuzzy coefficients. Fuzzy Optim Decis Mak 6:5-16. https://doi.org/10.1007/s10700-006-0021-0. }
$$

Montastruc L, Boix M, Pibouleau L, Azzaro-Pantel C, Domenech S (2013) On the flexibility of an eco-industrial park (EIP) for managing industrial water. $\mathrm{J}$ Clean Prod 43:1-11. https://doi.org/10.1016/j.jclepro.2012.12.039.

Olesen S G, Polley G T (1996) Dealing with plant geography and piping constraints in water network design. Process Saf Environ Protect 74:273-276. https://doi.org/10.1205/095758296528626.

Ramos M A, Boix M, Aussel D, Montastruc L, Domenech S (2016) Water integration in eco-industrial parks using a multi-leader-follower approach. Comput Chem Eng 87:190-207. https://doi.org/10.1016/j.compchemeng.2016.01.005.

Stancu-Minasian I M (2019) A ninth bibliography of fractional programming. Optimization 68:2123-2167. https://doi.org/10.1080/02331934.2019.1632250.

Tan Q, Huang G H, Cai Y P (2013) Multi-source multi-sector sustainable water supply under multiple uncertainties: an inexact fuzzy-stochastic quadratic programming approach. Water Resour Manag 27:451-473. https://doi.org/10.1007/s11269-012-0196-9.

Tan Q, Zhang T Y (2018) Robust fractional programming approach for improving agricultural water-use efficiency under uncertainty. J Hydrol 564:1110-1119. https://doi.org/10.1016/j.jhydrol.2018.07.080.

Tan R R, Foo D C Y, Manan Z A (2007) Assessing the sensitivity of water networks to noisy mass loads using Monte Carlo simulation. Comput Chem Eng 31:1355-1363. https://doi.org/10.1016/j.compchemeng.2006.11.005. 
Tan R R, Ng D K S, Foo D C Y (2010) Graphical approach to minimum flowrate targeting for partitioning water pretreatment units. Chem Eng Res Des 88:393-402. https://doi.org/10.1016/j.cherd.2009.09.004.

Taskhiri M S, Tan R R, Chiu A S F (2011) MILP model for emergy optimization in EIP water networks. Clean Technol Environ Policy 13:703-712. https://doi.org/10.1007/s10098-010-0341-1.

The People's Government of Tianjin Binhai New Area (2016) The special planning of water supply in the east of TEDA. http://www.tjbh.gov.cn/contents/6377/122537.html.

Tiu B T C, Cruz D E (2017) An MILP model for optimizing water exchanges in eco-industrial parks considering water quality. Resour Conserv Recycl 119:89-96. https://doi.org/10.1016/j.resconrec.2016.06.005.

Wada Y, Florke M, Hanasaki N, Eisner S, Fischer G, Tramberend S, Satoh Y, van Vliet M T H, Yillia P, Ringler C, Burek P, Wiberg D (2016) Modeling global water use for the 21st century: the water futures and solutions (WFaS) initiative and its approaches. Geosci Model Dev 9:175-222. https://doi.org/10.5194/gmd-9-175-2016.

Wang Y Y, Huang G H, Wang S, Li W (2016) A stochastic programming with imprecise probabilities model for planning water resources systems under multiple uncertainties. Stoch Environ Res Risk Assess 30:2169-2178. https://doi.org/10.1007/s00477-015-1134-1.

World Business Council for Sustainable Development (2006) Business in the world of water: WBCSD water scenarios to 2025. https://www.wbcsd.org/Programs/Food-and-Nature/Water/Resources/Bu siness-in-the-World-of-Water-WBCSD-water-scenarios-to-2025.

Xie Y L, Huang G H, Li W, Li J B, Li Y F (2013) An inexact two-stage stochastic programming model for water resources management in Nansihu Lake Basin, China. J Environ Manage 127:188-205. https://doi.org/10.1016/j.jenvman.2013.04.027.

Yoo C, Lee T Y, Mm J, Moon I, Jung J H, Han C, Oh J M, Lee I B (2007) Integrated water resource management through water reuse network design for clean production technology: State of the art. Korean Journal Of Chemical 24:567-576. https://doi.org/10.1007/s11814-007-0004-z.

Zhao J M, Li M, Guo P, Zhang C L, Tan Q (2017) Agricultural water productivity oriented water resources allocation based on the coordination of multiple factors. Water 9:1-22. https://doi.org/10.3390/w9070490. 
803

804

805

806

807

808

809

810

811

812

813

814

815

816

817

818

819

820 Subject to:

$821 \quad \sum_{j=1}^{n} a_{i j} x_{j}+\sum_{j=1}^{n} u_{i j} y_{j h} \leq b_{i}, \quad i=1,2, \mathrm{~L}, m ; h=1,2, \mathrm{~L}, v$

822

$\sum_{j=1}^{n} a_{k j} x_{j}+\sum_{j=1}^{n} u_{k j} y_{j h} \leq w_{k h}, \quad k=1,2, \mathrm{~L}, K ; h=1,2, \mathrm{~L}, v$

$823 \quad \sum_{h=1}^{v} p_{h}=1$

824

825

826 141:1-12. https://doi.org/10.1061/(ASCE)WR.1943-5452.0000470. https://doi.org/10.1016/j.jijepes.2013.05.022. https://doi.org/10.1016/j.apenergy.2013.07.053.

\section{Supplementary Material}

The two-stage linear fractional model can be formulated as follows:

$\max f=\frac{\sum_{j=1}^{n} c_{j} x_{j}+\sum_{h=1}^{v} \sum_{j=1}^{n} p_{h} e_{j} y_{j h}+\alpha}{\sum_{j=1}^{n} d_{j} x_{j}+\sum_{h=1}^{v} \sum_{j=1}^{n} p_{h} g_{j} y_{j h}+\beta}$

$x_{j} \geq 0, j=1,2, \mathrm{~L}, n$

$y_{j h} \geq 0, j=1,2, \mathrm{~L}, n ; h=1,2, \mathrm{~L}, v$

Zhou X, Huang G H, Zhu H, Yan B (2015) Two-stage chance-constrained fractional programming for sustainable water quality management under uncertainty. J Water Resour Plan Manage-ASCE

Zhou Y, Huang G, Zhu H, Li Z, Chen J P (2016) A factorial dual-objective rural environmental management model. J Clean Prod 124:204-216. https://doi.org/10.1016/j.jclepro.2016.02.081.

Zhu H, Huang G H (2011) SLFP: a stochastic linear fractional programming approach for sustainable waste management. Waste Manage 31:2612-2619. https://doi.org/10.1016/j.wasman.2011.08.009.

Zhu H, Huang G H (2013) Dynamic stochastic fractional programming for sustainable management of electric power systems. Int $\mathrm{J}$ Electr Power Energy Syst 53:553-563.

Zhu H, Huang W W, Huang G H (2014) Planning of regional energy systems: an inexact mixed-integer fractional programming model. Appl Energy 113:500-514.

\section{Appendix A. Solving procedure of two-stage linear fractional model}

If the denominator is strictly positive, this model can be transformed to: 
828 Subject to: $y_{j h}^{*} \geq 0, j=1,2, \mathrm{~L}, n ; h=1,2, \mathrm{~L}, v$

837 The above mentioned model could be solved by branch and bound algorithm directly.

Statements \& Declarations

\section{Funding}

This work was financially supported by the National Natural Science Foundation of China [52125902]; and Program for Guangdong Introducing Innovative and Entrepreneurial Teams [2019ZT08L213]. The authors are grateful to the editor and the anonymous reviewers for their insightful comments and suggestion.

\section{Competing Interests}

The authors have no relevant financial or non-financial interests to disclose.

\section{Author Contributions}

All authors contributed to the study conception and design. Material preparation, data collection and analysis were performed by Zhuoling Lan.The first draft of the 

versions of the manuscript. All authors read and approved the final manuscript. 
